\title{
Model Based Separation of Overlapping Latent Fingerprints
}

\author{
Qijun Zhao and Anil K. Jain, Fellow, IEEE
}

\begin{abstract}
Latent fingerprints lifted from crime scenes often contain overlapping prints, which are difficult to separate and match by state-of-the-art fingerprint matchers. A few methods have been proposed to separate overlapping fingerprints to enable fingerprint matchers to successfully match the component fingerprints. These methods are limited by the accuracy of the estimated orientation field, which is not reliable for poor quality overlapping latent fingerprints. In this paper, we improve the robustness of overlapping fingerprints separation, particularly for low quality images. Our algorithm reconstructs the orientation fields of component prints by modeling fingerprint orientation fields. In order to facilitate this, we utilize the orientation cues of component fingerprints, which are manually marked by fingerprint examiners. This additional markup is acceptable in forensics, where the first priority is to improve the latent matching accuracy. The effectiveness of the proposed method has been evaluated not only on simulated overlapping prints, but also on real overlapped latent fingerprint images. Compared with available methods, the proposed algorithm is more effective in separating poor quality overlapping fingerprints and enhancing the matching accuracy of overlapping fingerprints.
\end{abstract}

\section{INTRODUCTION}

Fingerprints are widely used for personal authentication in both forensic and civilian applications. Given a fingerprint image, fingerprint matchers extract features (e.g. minutiae) from it, and match the features against the reference feature templates to identify or verify the identity associated with the fingerprint [1]. Typically, the input image contains only a single fingerprint. However, in practice, particularly in forensics, two or more fingerprints could overlay on top of each other, resulting in an overlapped fingerprint image (see Fig. 1(a)). Latent fingerprints lifted from crime scenes may contain overlapping fingerprints, and live-scan fingerprint images sometimes also have multiple impressions of fingers because of the residual fingerprints left on the sensor. Such overlapped fingerprint images, while difficult to process, are useful forensic evidence for identifying suspects. Available fingerprint matchers, however, can not accurately match overlapping fingerprints, because they assume that a fingerprint image contains only a single fingerprint and hence single orientation field [2]. Our interest here is to develop algorithms to separate overlapping latents that will serve as a valuable tool in forensics. Note that in forensics, the matching accuracy of latents is extremely critical even if it involves some degree of manual intervention by latent examiners, including manual markup.

The prevailing procedure to recognize component fingerprints in an overlapped image is for latent examiners to

Qijun Zhao and Anil K. Jain are with the Department of Computer Science and Engineering, Michigan State University, East Lansing, MI, 48824 USA. Anil K. Jain is also with the Department of Brain \& Cognitive Engineering, Korea University, Anamdong, Seongbukgu, Seoul 136-713, Republic of Korea. E-mail: \{qjzhao, jain\}@cse.msu.edu.

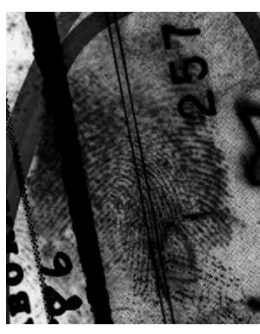

(a)

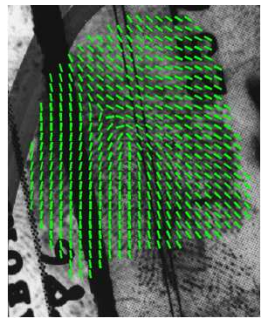

(d)

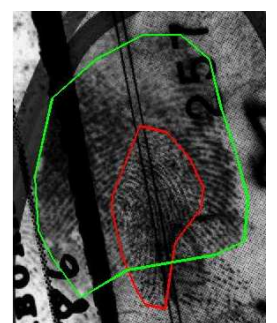

(b)

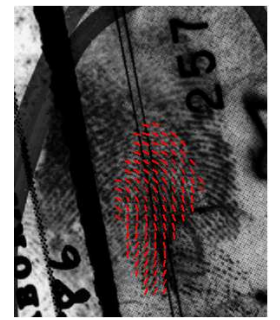

(e)

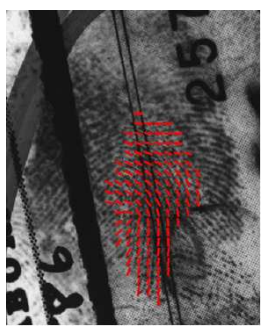

(c)

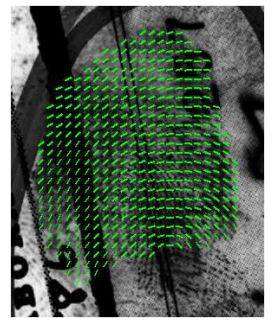

(f)
Fig. 1. A fingerprint image containing two overlapping latent prints (a) constructed by overlaying two latents from the NIST SD27 database [3]. The individual latents are marked as red and green polygons in (b). The two component orientation fields separated by using state-of-the-art relaxation labeling based method [4] are shown in (c) and (d). The orientation fields reconstructed by the proposed method are shown in (e) and (f). A commercial off the shelf (COTS) matcher was used to match the two component fingerprints with their corresponding mated rolled fingerprints. The match scores are both 0 without separation (using the overlapped image in (a)), and are improved to 6 and 1 for (c) and (d), respectively. By using the proposed method, the match scores are further improved to 29 and 3 for (e) and (f), respectively, showing the superior performance of the proposed method.

manually mark minutiae for each component fingerprint in the image, and then feed this information to a fingerprint matcher. Manually marking minutiae in overlapped fingerprint images is not only tedious, but also very difficult. Recent publications [2], [4]-[7] have proposed algorithms to separate two overlapping fingerprints with minimal markup. Given the component fingerprints, latent examiners no longer need to mark the minutiae since the component fingerprints can be effectively matched by commercial matchers. Chen et al. [2] and Shi et al. [4] showed an improvement in the matching accuracy of component fingerprints compared to the overlapping print by applying a relaxation labeling based algorithm on relatively good quality overlapped images synthesized from the FVC2002 Db1_b database [8]. Figure 2 shows an example of synthesized overlapped fingerprint image used in [2] and [4].

State-of-the-art overlapping fingerprints separation algorithms [2], [4] consist of three main steps. First, orientation field is estimated from the overlapped fingerprint image by local Fourier analysis. Second, the mixed orientations in the overlapping area are separated into two components, one for 


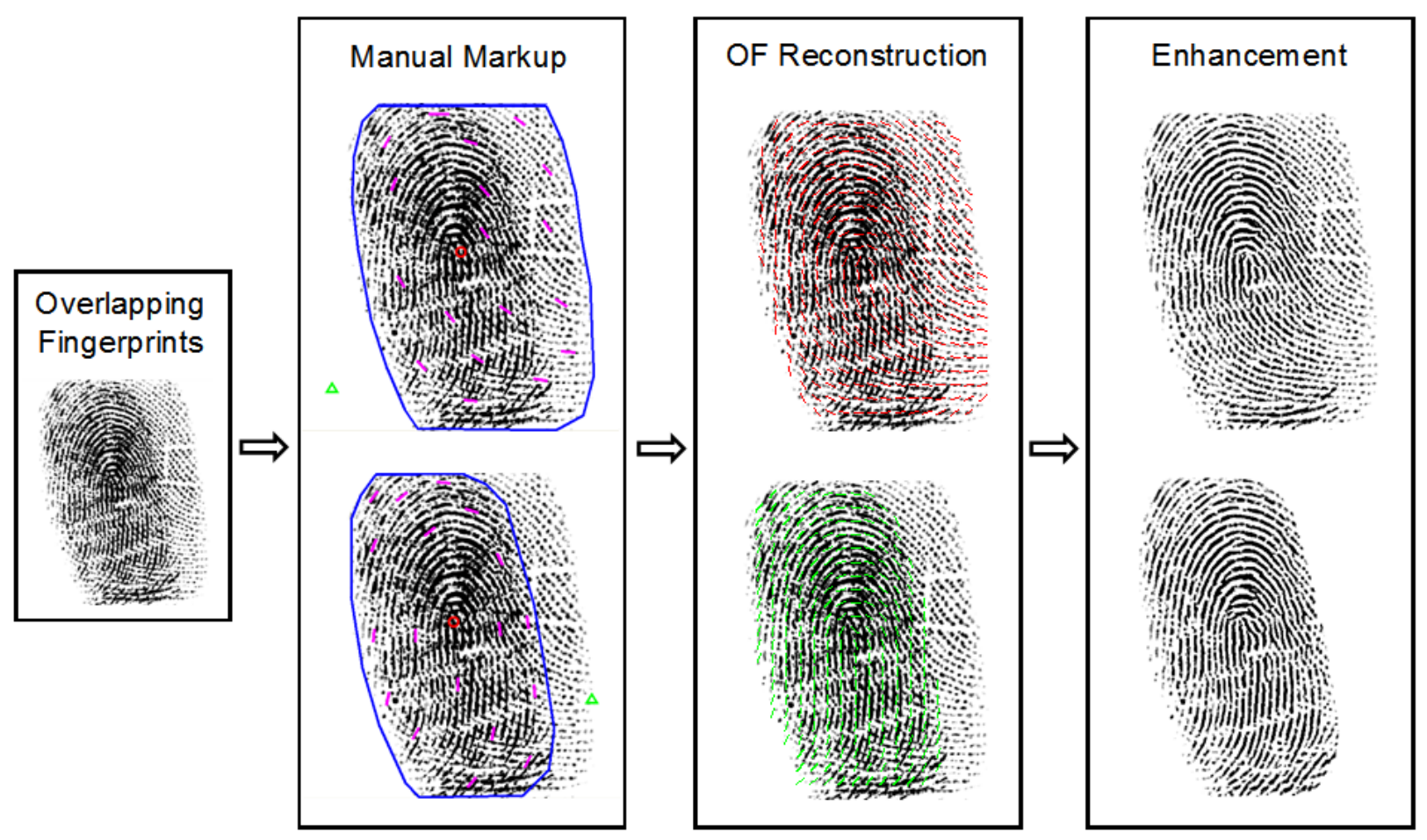

Fig. 2. Processing steps in the proposed overlapping fingerprint separation algorithm. (i) Manual markup of regions of interest (represented by polygons), singular points (cores represented by circles and deltas by triangles), and orientation cues (represented by magenta strokes), (ii) orientation field (OF) reconstruction, and (iii) enhancement. The best match scores between the two component fingerprints in this overlapped fingerprint image and their mated template fingerprints by a COTS matcher are, respectively, 38 and 68 without separation, 140 and 47 after separation by the algorithm in [4], and 328 and 128 after separation by the proposed algorithm. The overlapped image is from the database used in [2] and [4].

each component fingerprint, by using a relaxation labeling method. Third, given the orientation fields of the component fingerprints, the two overlapping fingerprints are separated by enhancing the overlapped fingerprint image with Gabor filters that are tuned according to the orientation fields of component fingerprints. For such an approach to be effective, it is critical to obtain accurate orientation fields of the component fingerprints. The initial estimation of mixed orientation field (from the overlapping print) is thus a bottleneck in these relaxation labeling based methods. For poor quality overlapped latent images, it is very difficult to automatically esitmate ridge orientations. Comparing the overlapped latent image in Fig. 1(a) and the overlapped image in Fig. 2, one can easily see the challengs in separating low quality overlapping latents caused by complicated background and unclear ridge structures in the images. Another drawback of the relaxation labeling based methods is that they separate the mixed orientation fields based only on local ridge orientation consistency under the assumption that fingerprint ridge orientation fields are locally smooth. Figure 1 shows the separated orientation fields of an overlapped latent image by using the method in [4] (Figs. 1(c) and 1(d)) and the method proposed in this paper (Figs. 1(e) and 1(f)). As can be seen, the poor quality of latent images seriously degrades the performance of the relaxation labeling based method in [4].

The objective of this paper is to improve the robustness of overlapping fingerprint separation, particularly for poor quality images (e.g. overlapping latents). Previous work on this topic [2], [4] mainly focused on relatively good quality overlapping fingerprints and showed improvement in matching accuracy only on simulated overlapping live-scan fingerprints. In this paper, instead of separating the estimated mixed orientation field, we reconstruct the orientation fields of component fingerprints via modeling orientation fields and then predicting unknown orientation fields based on a small number of manually marked orientation cues in fingerprints. The fingerprint orientation field model not only ensures the local smoothness of component orientation fields, but also serves as a global constraint on the reconstructed orientation fields. For these reasons, the proposed model based method significantly improves the accuracy of overlapping fingerprints separation, especially for the practical scenario of poor quality overlapped latent images.

The rest of this paper is organized as follows. Section II gives an overview of the proposed separation algorithm. Section III introduces in detail the reconstruction of orientation fields of component fingerprints via orientation field modeling and prediction. Section IV then presents experimental evaluation results along with some discussion. Section V finally concludes the paper.

\section{Overview of Separation Algorithm}

The flowchart of the proposed overlapping fingerprint separation algorithm is shown in Fig. 2. To separate two overlapping fingerprints, region of interest (or ROI), singular points (core and delta), and a small number of orientation cues 
(represented by strokes) are provided for each fingerprint. ROI masks out the regions of the individual components in overlapped image. Singular points and orientation cues help identify the ridge patterns of overlapping fingerprints. Singular points that are outside of the ROI and not visible in the image might also be marked with the best guess of their positions. Such singular points are shown in green color in Fig. 2. Orientation cues specify the ridge orientations in some of the local blocks of a fingerprint, which together with singular points (if any) are used to estimate the parameters of fingerprint orientation field model. Given these model parameters, orientation estimates are more likely to be accurately predicted for the fingerprint blocks. Again, these manual markups, much simpler than marking all the minutiae points, ensure accurate separation of component fingerprints that is crucial for latent fingerprint analysis. In the next section, we will introduce in detail the procedure of orientation field modeling and prediction for reconstructing orientation fields (OF) of overlapping fingerprints. Given the reconstructed orientation fields of the two component fingerprints, the ridge frequency in the two fingerprints can be estimated by using the method in [9]. Finally, the two overlapping fingerprints are separated by enhancing the overlapped fingerprint image with Gabor filters that are tuned to their respective orientation fields and ridge frequencies [9]. This last step is similar to the methods in [2] and [4].

Compared with the relaxation labeling based methods in [2] and [4], the proposed algorithm does require additional inputs, namely singular points and orientation cues. In [2] the authors also made an attempt to incorporate manually marked singular points into their relaxation labeling based separation method without consistent performance improvement. Chen et al. [2] subtracted the singular orientation field generated by the manually marked singular points according to the ZeroPole model [10] from the intial mixed orientation fields. The resulting orientation fields were assumed to be continuous and the relaxation labeling method was applied to separate these continuous orientation fields. There are two potential problems with their approach. (i) It is unknown which component orientation is affected by which singular points before separation. Consequently, all possible combinations have to be evaluated, which makes the method very complicated and error-prone. (ii) Errors in the initial mixed orientation fields can significantly affect the separation results.

The relaxation labeling based method of Shi et al. [4], an improvement over [2], still suffers from the errors in the initial mixed orientation fields, which can be serious when using local Fourier analysis to automatically extract orientations from poor quality overlapped latents (see Fig. 1). Although fewer manual markup inputs (or even fully automatic systems) can reduce the burden on fingerprint examiners and help to improve the throughput, it is more desirable in practice to have higher recognition accuracy with acceptable manual intervention. Moreover, it is a common practice in latent examination to manually mark singular points [11], and it is much easier for fingerprint examiners to trace a small number of reliable ridge segments than to mark all minutiae in a latent. These facts motivated us to propose the separation method in this

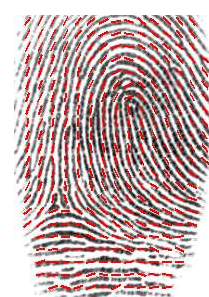

(a)

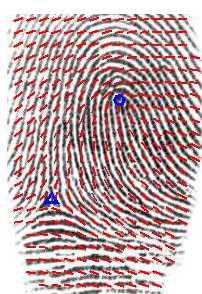

(b)

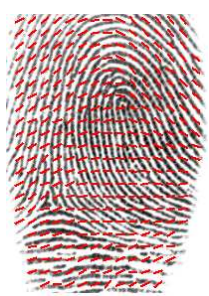

(c)
Fig. 3. Fingerprint orientation field. (a) A fingerprint image in FVC2002 Db1-a [8], and its orientation field estimated by gradient based method [1], which is generally smooth except at the regions around singular points. (b) Orientation field generated by the singular points according to the Zero-Pole model [10]. (c) Residual orientation field, which is the difference between the orientation fields in (a) and (b); it is smooth and continuous.

paper in order to effectively utilize the relatively simple cues of component fingerprints provided by fingerprint examiners. The proposed model-based method permits an effective use of the manual markup.

\section{ORientation Field Modeling And Prediction}

\section{A. Orientation Field Models}

The orientation field of a fingerprint represents the dominant local ridge orientations. Let $\Omega$ denote the region of interest in the input overlapping fingerprint. The fingerprint orientation field can be then viewed as a function $\theta(x, y)$ of the location $(x, y) \in \Omega$, where $\theta(x, y) \in[0, \pi)$ represents the dominant local ridge orientation at $(x, y)$. It is, however, difficult to directly model the fingerprint orientation field because of the intrinsic orientation discontinuity [1]. This problem can be solved by using doubled orientations $2 \theta(x, y)$ and modeling it in the cosine and sine planes (or the complex plane) [12].

The fingerprint orientation field $\theta(x, y)$ is smooth in most locations of $\Omega$, except in the regions around singular points (see Fig. 3(a)). The Zero-Pole model [10] for the orientation field generated by singular points is defined as

$$
\theta^{S P}(z)=\frac{1}{2} \arg \left(\frac{\prod_{i=1}^{K}\left(z-z_{c_{i}}\right)}{\prod_{j=1}^{L}\left(z-z_{d_{j}}\right)}\right),
$$

where $z=x+i \cdot y$ is a point in the fingerprint represented in the complex plane, $z_{c_{i}}$ and $z_{d_{j}}$ correspond, respectively, to the $i^{\text {th }}$ core and the $j^{\text {th }}$ delta, and $K$ and $L$ are the numbers of cores and deltas in the fingerprint. In this model, cores are taken as zeros and deltas as poles in the complex plane, and the orientation at a point $z$ is determined by the sum of influence of all cores and deltas. The Zero-Pole model provides an effective tool for describing the contribution of singular points to fingerprint orientation field. However, it is not able to accurately describe the orientation field of many real fingerprints (see Fig. 3(b)). Fingerprints with the same singular points can have different orientation fields [13].

In order to more accurately approximate the fingerprint orientation field, Zhou et al. [13] proposed a combination of a point-charge model [14] and a polynomial model [14]. The point-charge model, similar to the Zero-Pole model, describes the orientation field close to singular points and the polynomial 
model approximates the orientation field in the rest part of fingerprint, which is supposed to be smooth and continous. The main idea of the polynomial model is to approximate the cosine and sine components of doubled orientations by using a linear combination of a set of basis functions. In [13], monomials were used as the basis functions. Some of the other basis functions that have been explored include Legendre polynomials [15] and 2D Fourier series [16].

Motivated by the combination model in [13] and its successful application for latent fingerprint enhancement [17] and fingerprint reconstruction [18], we first remove the influence of singular points from the orientation field by subtracting $\theta^{S P}$ from $\theta$, and then approximate the residual orientation field with a set of basis functions. Let

$$
\theta^{R}=\theta-\theta^{S P}
$$

be the residual orientation field (see Fig. 3(c)), and $\left\{\phi_{i}(x, y) \mid i=0,1, \cdots, n\right\}$ the set of basis functions. The cosine and sine components of the doubled residual orientations can be approximated by

$$
\left\{\begin{array}{l}
\cos \left(2 \theta^{R}(x, y)\right)=\sum_{i=0}^{n} a_{i} \phi_{i}(x, y), \\
\sin \left(2 \theta^{R}(x, y)\right)=\sum_{i=0}^{n} b_{i} \phi_{i}(x, y)
\end{array}\right.
$$

where $\left\{a_{i}, b_{i} \mid i=0,1, \cdots, n\right\}$ are the coefficients of the model. Given the residual orientation field in the region of interest $\left\{(x, y)_{j} \in \Omega \mid j=1,2, \cdots, m\right\}$, the coefficients can be obtained by solving the following minimization problems using least squares optimization:

$$
\left\{\begin{array}{l}
\hat{\mathbf{a}}=\arg \min _{\mathbf{a}}\|\Phi \cdot \mathbf{a}-\mathbf{C}\|^{2}, \\
\hat{\mathbf{b}}=\arg \min _{\mathbf{b}}\|\Phi \cdot \mathbf{b}-\mathbf{S}\|^{2},
\end{array}\right.
$$

where $\mathbf{a}=\left(a_{0}, a_{1}, \cdots, a_{n}\right)^{T}, \mathbf{b}=\left(b_{0}, b_{1}, \cdots, b_{n}\right)^{T}$,

$$
\begin{gathered}
\mathbf{C}=\left(\begin{array}{c}
\cos \left(2 \theta^{R}\left((x, y)_{1}\right)\right) \\
\cos \left(2 \theta^{R}\left((x, y)_{2}\right)\right) \\
\vdots \\
\cos \left(2 \theta^{R}\left((x, y)_{m}\right)\right)
\end{array}\right), \mathbf{S}=\left(\begin{array}{c}
\sin \left(2 \theta^{R}\left((x, y)_{1}\right)\right) \\
\sin \left(2 \theta^{R}\left((x, y)_{2}\right)\right) \\
\vdots \\
\sin \left(2 \theta^{R}\left((x, y)_{m}\right)\right)
\end{array}\right) \\
\Phi=\left(\begin{array}{cccc}
\phi_{0}\left((x, y)_{1}\right) & \phi_{1}\left((x, y)_{1}\right) & \cdots & \phi_{n}\left((x, y)_{1}\right) \\
\phi_{0}\left((x, y)_{2}\right) & \phi_{1}\left((x, y)_{2}\right) & \cdots & \phi_{n}\left((x, y)_{2}\right) \\
\vdots & \vdots & \ddots & \vdots \\
\phi_{0}\left((x, y)_{m}\right) & \phi_{1}\left((x, y)_{m}\right) & \cdots & \phi_{n}\left((x, y)_{m}\right)
\end{array}\right)
\end{gathered}
$$

and $T$ is the transpose operator.

\section{B. Orientation Field Reconstruction}

For orientation field prediction and reconstruction, only the orientations at a subset of $\Omega$ are assumed to be known (given cues); the orientations at remaining locations of $\Omega$ are estimated based on the model. Let us denote these two subsets as $\Omega_{C}$ and $\Omega_{X}\left(\Omega=\Omega_{C} \cup \Omega_{X}\right)$, respectively. For the overlapping fingerprints separation problem, $\Omega_{C}$ corresponds to the blocks where local orientation cues are given, and $\Omega_{X}$ is the set of other locations in the component fingerprint.

Given a fingerprint orientation field model, unknown orientations are predicted as follows: (i) compute the model coefficients based on the known orientations in $\Omega_{C}$, and (ii) use the established model to estimate the orientations in $\Omega_{X}$.
More specifically, given the ROI, singular points, and the orientation cues in $\Omega_{C}$, the model coefficients are computed according to (4). The residual orientation at $(x, y)_{j} \in \Omega_{X}$ ( $j=1,2, \cdots, m_{X}$, where $m_{X}$ is the total number of pixels or blocks in $\Omega_{X}$ ) can be then estimated as

$$
\hat{\theta}^{R}\left((x, y)_{j}\right)=\frac{1}{2} \arctan \left(\frac{\sum_{i=0}^{n} \hat{b}_{i} \phi_{i}\left((x, y)_{j}\right)}{\sum_{i=0}^{n} \hat{a}_{i} \phi_{i}\left((x, y)_{j}\right)}\right) .
$$

Finally, the orientation at $(x, y)_{j} \in \Omega_{X}$ is obtained by adding back the influence of singular points to the estimated residual orientation, i.e.

$$
\hat{\theta}\left((x, y)_{j}\right)=\hat{\theta}^{R}\left((x, y)_{j}\right)+\theta^{S P}\left((x, y)_{j}\right) .
$$

The above reconstructed orientation field is, however, still not very accurate (note the upper left corner and the region around the core in Fig. 4(b)). To obtain a more accurate reconstruction, instead of predicting all the unknown orientations at once (one shot approach), we first predict the locations in the neighborhood of $\Omega_{C}$ and then gradually predict the orientations at farther locations from $\Omega_{C}$ based on both the known orientations in $\Omega_{C}$ and the previously predicted orientations. The orientation field model parameters keep getting updated during the iterative process. At each iteration, the known orientations in $\Omega_{C}$ and the previously predicted orientations are regularized by substituting them with the output of the last updated model. Such regularization can gradually correct the errors in the given cues in $\Omega_{C}$ and previous predictions. Algorithm 1 summarizes the approach, and Fig. 4 shows the results on an example overlapped fingerprint image. The orientation field reconstructed by the proposed iterative algorithm is more accurate than that by one-shot prediction. A similar iterative approach was also employed in the so-called "Smooth Extensions" orientation field reconstruction algorithm in [19]. Our algorithm differs from "Smooth Extensions" in that (i) we explicitly consider singular points and (ii) we incorporate regularization into the reconstruction process.

\section{Related Work}

Orientation field modeling and reconstruction are fundamental problems in many fingerprint related applications, such as fingerprint ridge orientation extraction [13], [14], fingerprint image enhancement [17], fingerprint image reconstruction [18], [20], [21], and fingerprint matching [11], [19]. A number of different fingerprint orientation field models have been proposed in the literature. Some of them describe the orientation fields generated by singular points. These models, including the Zero-Pole model [10] and its variants [22], [23], can not handle the arch fingerprints which do not have any singular points or accurately approximate the orientation fields far from singular points [14]. This limitation inspired the more elaborate models in [13], [24]-[26]. In [13], the orientation fields nearby and far from singular points are described, respectively, by a point-charge model and a polynomial model, and the two models are combined by weighted summation. In [24] and [25], fingerprints are also divided into several different regions according to the singular points. Orientation fields in these 


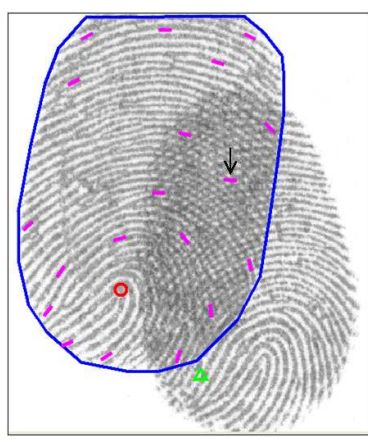

(a)

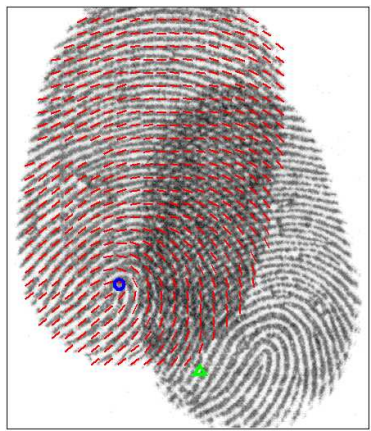

(e)

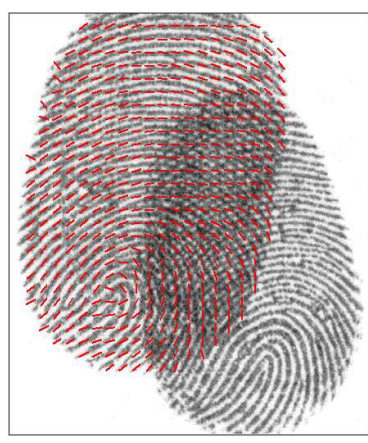

(b)

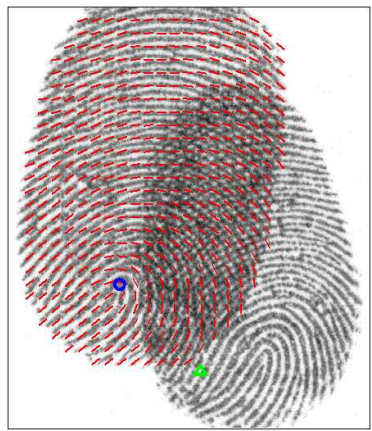

(f)

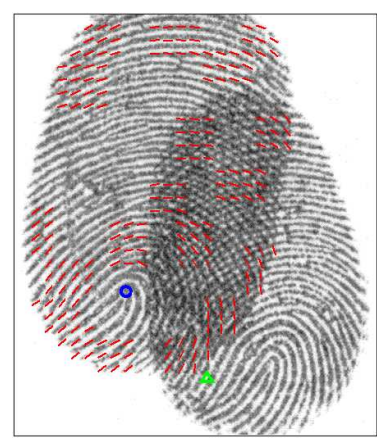

(c)

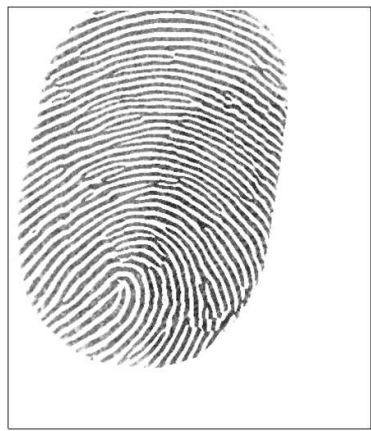

(g)

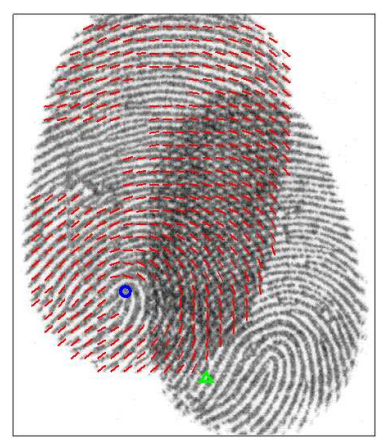

(d)

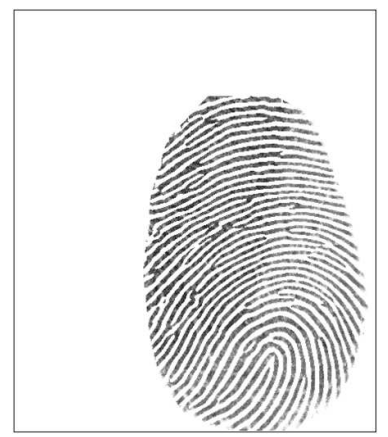

(h)

Fig. 4. Orientation field reconstruction. (a) An overlapped image along with the ROI, singular points, and orientation cues in one of the two component fingerprints. The arrow in (a) points to a cue that is not very accurate. (b)-(f) show the reconstructed orientation fields of that component fingerprint. (b) obtained by one-shot prediction. (c)-(f) results at the first, second, third and final iterations by using the proposed iterative approach. Note that the erroneous input cues and predictions are gradually corrected. $(\mathrm{g})$ and $(\mathrm{h})$ are the two separated component fingerprints of (a) based on the reconstructed orientation fields by the proposed method.

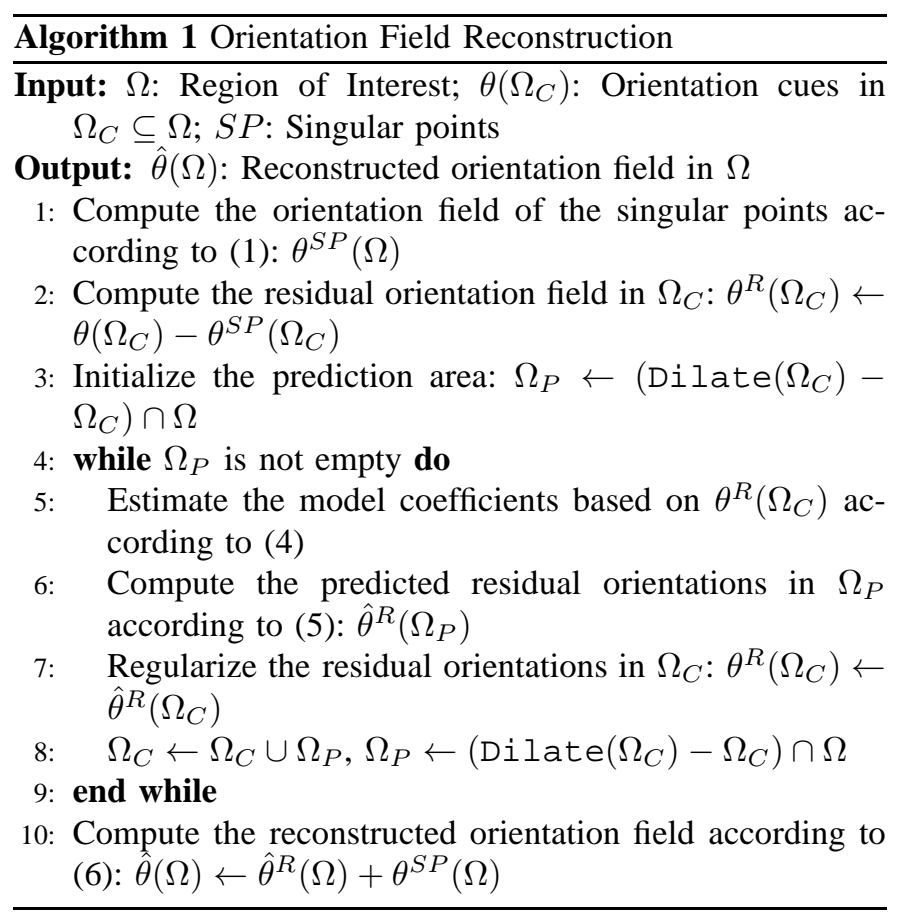

regions are then approximated by using piecewise linear phase portaits. Both these models require the partition of fingerprints into predefined regions, which is, however, very difficult in practice, especially for latent fingerprints, which are partial and often do not contain any singular points. Huckemann et al. [26] proposed to use quadratic differentials to model the orientation fields in different types of fingerprints. To apply the quadratic differential based models, the fingerprints have to be aligned with respect to the coordinate systems defined by singular points (or points with highest curvatures in arch fingerprints). However, it is not feasible to establish the required coordinate systems when some of the singular points are missing, as in often the case with latent fingerprints.

The combination model initially proposed in [13] was recently improved by Feng et al. [18] for the purpose of reconstructing orientation fields from a given set of minutiae. Instead of dividing fingerprints into different parts, Feng et al. divided the fingerprint orientation fields into singular and continuous components, and approximated them by using the Zero-Pole and polynomial models, respectively. In other words, the Zero-Pole model describes the influence of singular points (if any) on the whole fingerprint orientation field, and the polynomial model approximates the residual orientation field. In [18], it was shown that this improved combination model performs better than the interpolation model in [20] and the adapted Zero-Pole model in [21]. This model has also been successfully applied to latent fingerprint enhancement in [17]. Therefore, in this paper, we employ this combination model. Unlike in [17] and [18], we apply the model in an iterative approach to get more accurate prediction of unknown orientations (see Fig. 4).

In the polynomial models, a set of basis functions are 
needed to represent the fingerprint orientation fields. While monomials were used by [17] and [18], Ram et al. [15] and Wang et al. [16] proposed to use Legendre polynomials and trigonometric polynomials, both of which compose orthogonal bases and are claimed to be effective in approximating fingerprint orientation fields without prior knowledge of singular points. Recently, Wang et al. [19] applied the trigonometric polynomial based model (called FOMFE) to reconstruct the full orientation fields from partial fingerprints. Again, they did not explore singular points in the reconstruction process. Some other orientation field models which do not require prior knowledge of singular points include the probability based models in [27] and [28], which have rather high computational complexity. In this paper, we will compare the performance of different polynomial based models for both cases: using singular points and not using singular points.

\section{Evaluation AND Discussion}

\section{A. Separation Performance}

There is no public domain database of overlapping latent fingerprints. It is also difficult to obtain such images from the forensics laboratories because we have been told that, after manual separation, the overlapped prints are not kept in the database. We, therefore, report our results on the following three scenarios.

- Simulated overlapping livescan fingerprints

- Simulated overlapping latent fingerprints

- Real overlapping latent fingerprints

See Table I for details of the three databases. Note that the database of simulated overlapping livescan fingerprints was also used in [2] and [4]. But they did not test the matching performance of overlapping latents (i.e. the second and third scenarios). The performance and comparison of separation algorithms is based on the matching results between the separated component fingerprints and the enrolled full fingerprints. In the following experiments, we use up to fourth order monomials [13] as the basis functions for the proposed algorithm, and compare the proposed algorithm with the relaxation labeling approach in [4]. Two state-of-the-art COTS fingerprint matchers are employed, referred to as COTS 1 and COTS2.

1) Simulated Overlapping Livescan Fingerprints: We first compare the performance of the proposed model based algorithm with the relaxation labeling based algorithm in [4] on the database used in [2] and [4]. This database contains 100 overlapped fingerprint images, which were synthesized from the images in FVC2002 Db1-b [8]. Figure 2 shows an example overlapped fingerprint image in this database. Images in this database are livescan fingerprint images, which generally have better quality than latent images. In the experiments, the component fingerprints are matched against a background database of 60 images from FVC2002 Db1-b and 800 images from FVC2002 Db1-a. Each component fingerprint has six mates in the background database (the images used for synthesizing overlapping fingerprints are excluded), and the maximum match score between the component fingerprint and its mates is taken as its final score. Figure 5 shows

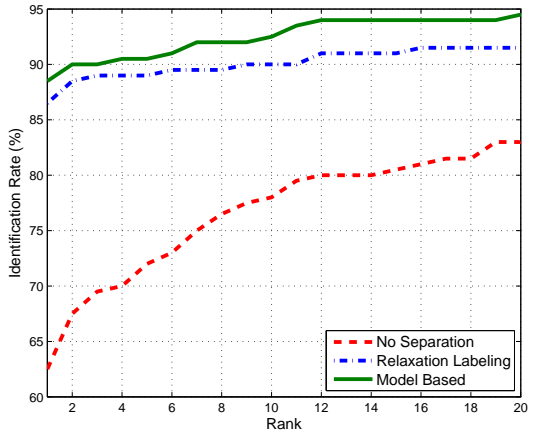

(a)

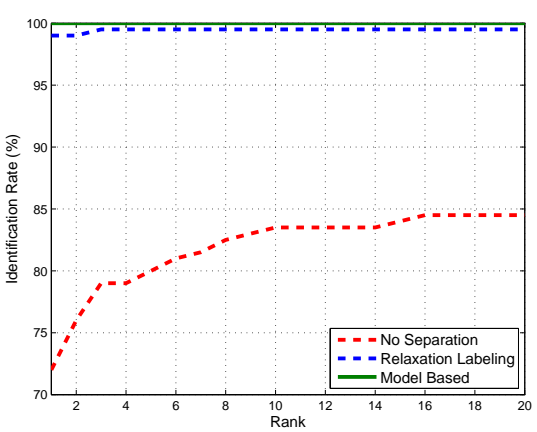

(b)

Fig. 5. CMC curves of (a) COTS1 and (b) COTS2 matchers on the simulated overlapping fingerprints in the database used in [2], [4]. In the case "No Separation", the component fingerprints in the original overlapped fingerprint images are directly matched to full prints; otherwise, they are first separated by using different algorithms and then matched.

the cumulative match characteristic (CMC, Identification Rate vs. Rank) curves for COTS1 and COTS2 matchers on the component fingerprints separated by different algorithms. As can be seen from these curves, after separating the component fingerprints, both the matchers can more accurately identify the fingerprints, compared to not separating the overlapped prints. Further, the accuracy improvement is higher by using the proposed separation algorithm compared to [4] (note that the COTS2 matcher we use in this paper has much better matching accuracy compared to the matcher used in previous studies [4] and [2]). Owing to the relatively good quality of these images, the rank-1 identification rate of COTS2 is improved from $72 \%$ (no separation) to $99 \%$ (using the relaxation labeling based algorithm in [4]) and 100\% (using the proposed model based algorithm). Figure 6 shows two component fingerprints, which are both correctly identified at rank-1 by COTS2 after being separated by the proposed method, and at rank-31 and rank-3, respectively, after being separated by the relaxation labeling based method.

2) Simulated Overlapping Latents: In order to evaluate the performance of separation algorithms on low quality latent images, we construct a set of fifteen simulated overlapping latents by using the latent fingerprint images in NIST SD27 [3]. Authors of [2] synthesized overlapping livescan fingerprints by first normalizing the gray-scale of two livescan fingerprint images and then taking the minimum gray value 
TABLE I

THREE DATABASES USED IN EXPERIMENTAL EVALUATION.

\begin{tabular}{|l|l|l|l|}
\hline Database & Image Type & Source & \# Images \\
\hline \hline 1 & Simulated overlapping livescan fingerprints & FVC2002 Db1-b [8] & 100 \\
\hline 2 & Simulated overlapping latent fingerprints & NIST SD27 [3] & 15 \\
\hline 3 & Real overlapping latent fingerprints & Forensics lab & 4 \\
\hline
\end{tabular}

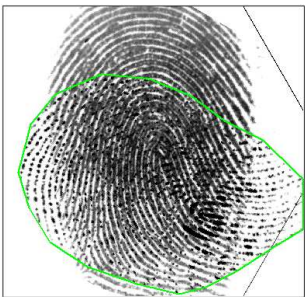

(a)

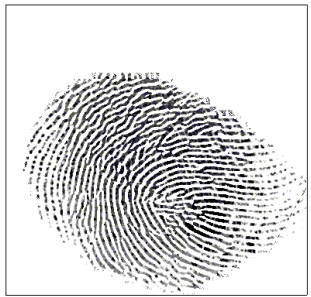

(c)

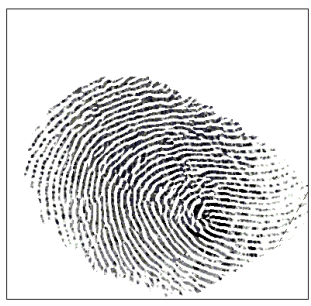

(e)

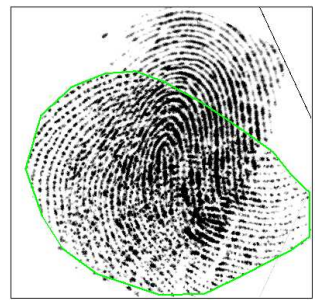

(b)

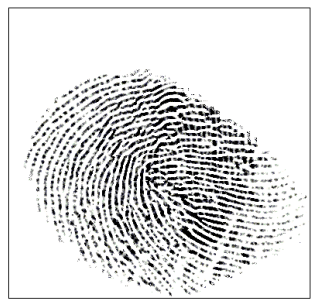

(d)

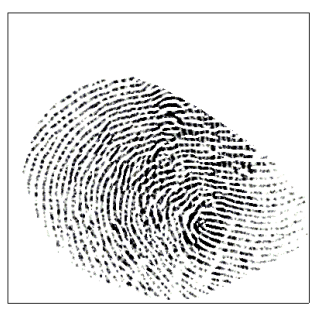

(f)
Fig. 6. Two component fingerprints, as marked by green polygons, in the database of simulated livescan overlapping fingerprints. They are correctly identified by COTS 2 matcher at rank- 1 after being separated by the proposed model based method, and at rank-31 and rank-3 after being separated by the relaxation labeling based method. (a-b) show the overlapped fingerprint images, (c-d) are the component fingerprints separated by the relaxation labeling based method, and (e-f) are the component fingerprints separated by the proposed method.

between corresponding pixels in the two images. However, this approach does not work well for latent images because latent images usually have very complicated background and different latents can have very different gray-scale ranges (see Fig. 7). Thus, we synthesize overlapping latents in the following way: (i) normalize the gray-scale of original latent images using histogram equalization, (ii) set the pixel values in the simulated overlapped latent image as the average of the intensities of the corresponding pixels in the two component latents (which are assumed to have the same size), (iii) and post-process the resulting overlapped latent image using histogram equalization.

The component latent fingerprints in these simulated overlapped latent images are matched against a background database consisting of 258 rolled fingerprints from NIST SD27

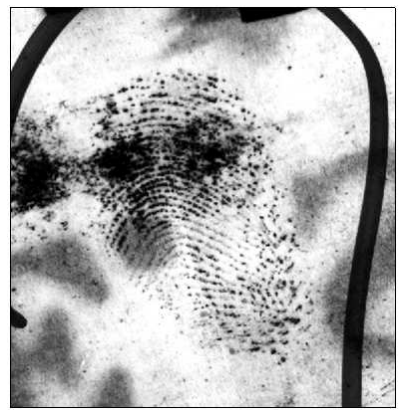

(a)

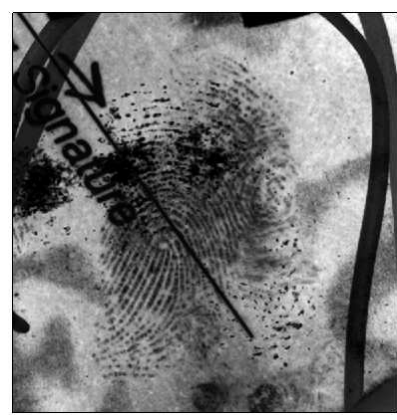

(c)

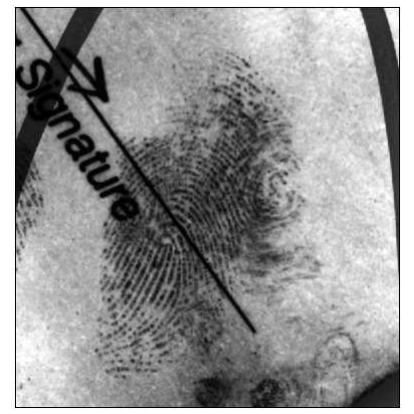

(b)

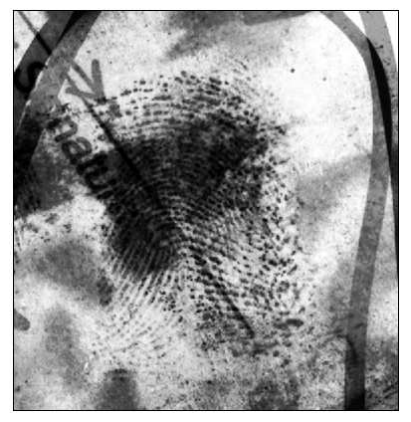

(d)
Fig. 7. Synthesizing overlapping latents. (a) and (b) are two source latent images from NIST SD27 [3], (c) simulated overlapped latent image by using the approach in [2] and [4], and (d) simulated overlapped latent image by using the approach in this paper.

and the first 27,000 rolled fingerprints from NIST SD14 [29]. The identification results of COTS1 and COTS2 matchers are presented in Fig. 8 and Fig. 9, respectively. By comparing the retrieval ranks of the mated full fingerprints of the component latents before and after separation, we can see that while the relaxation labeling based method can improve the matching ranks of 15 of the 30 component latents for COTS1 matcher and 17 of the 30 component latents for COTS2 matcher, the proposed model based method can improve the ranks of 22 of the component latents for both COTS1 and COTS2 matchers. This shows the superiority of the proposed method over the relaxation labeling based method for separating low quality overlapping latents. Figure 10 shows the separated component latents in the simulated overlapped latent image in Fig. 7. Note the poor performance of the relaxation labeling based method in the relatively dark area in the image and in the area around singular points. Using COTS2 matcher, the ranks of the mated full prints of the two original component latents are 4,544 and 24,859, respectively. After separating them by the relaxation labeling based method, COTS2 matcher can identify the second component latent at an improved rank of 16,180, 


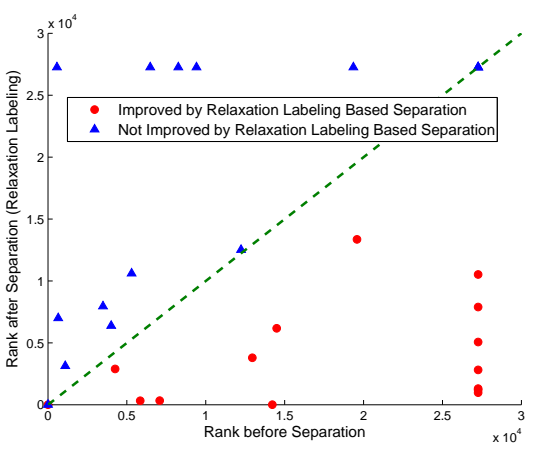

(a)

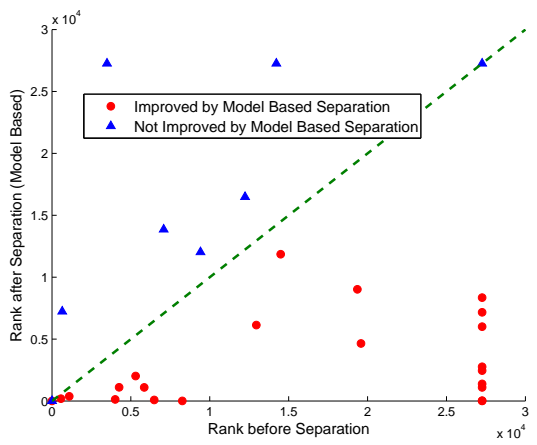

(b)

Fig. 8. Identification results of COTS1 matcher on the simulated overlapping latents.

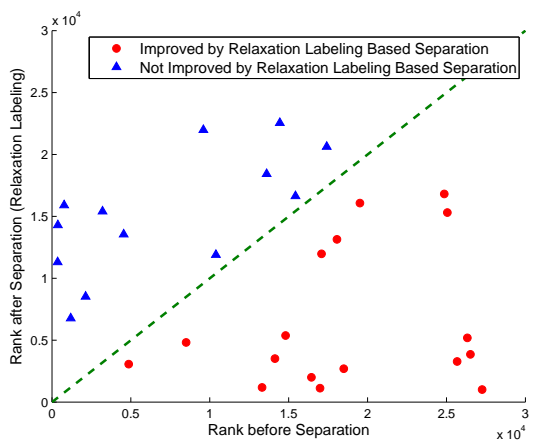

(a)

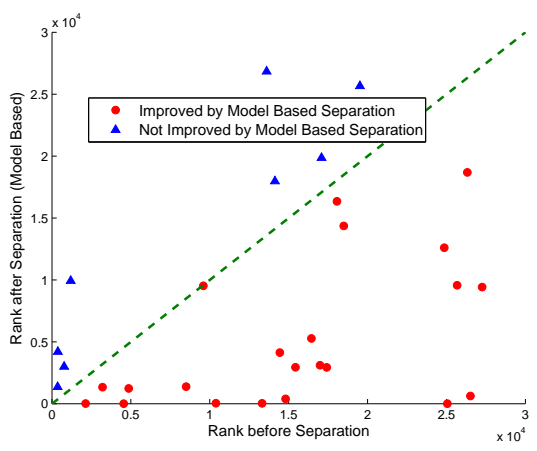

(b)

Fig. 9. Identification results of COTS2 matcher on the simulated overlapping latents.

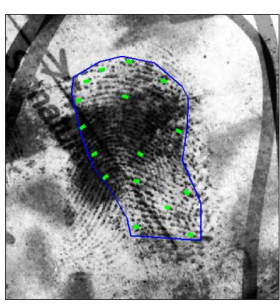

(a)

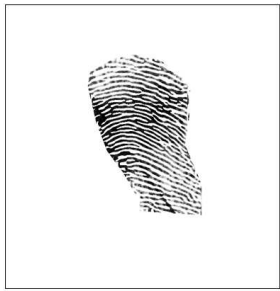

(c)

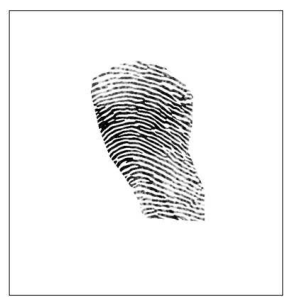

(e)

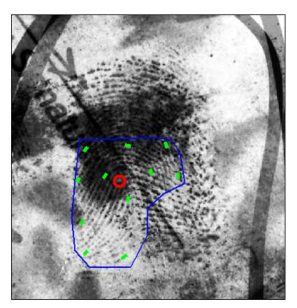

(b)

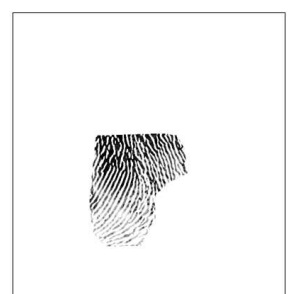

(d)

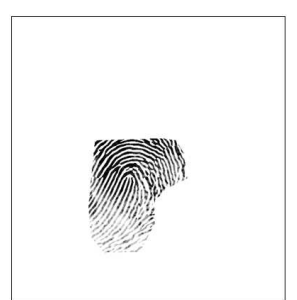

(f)
Fig. 10. Separated component latents in the simulated overlapped latent image in Fig. 7. (a-b) manually marked features of the two component latents, (c-d) component fingerprints separated by the relaxation labeling based method, and (e-f) component fingerprints separated by the proposed method. Using COTS2 matcher, the ranks of the two component latents are improved from 4,544 and 24,859 to 1 and 12,602 , respectively, after being separated by the proposed method. The corresponding ranks after being separated by the relaxation labeling based method are 13,543 and 16,810 , respectively.

but the first component latent at a higher rank of 13,543. In contrast, by applying the proposed model based separation method, the first component latent can be correctly identified by COTS 2 matcher at rank-1, and the rank of the mated full print of the second component latent is also improved to 12,602 .

3) Real Overlapping Latents: We have collected four images of real overlapping latents from two donors (one male and one female) with cooperation from Michigan State Forensics Laboratory. See Fig. 11. Latents in Figs. 11(a) and 11(b) have relatively simple background, while latents in Figs. 11(c) and 11(d) have complicated background with many letters, which severely degrades the quality of the latent fingerprints. In all these four images, the overlapping area comprises a large portion of the component latent fingerprints, and few discriminative features (e.g. minutiae) are available in the nonoverlapping area. As a result, it is of significant importance to recover the overlapping area of the component fingerprints to identify the latents.

The separated component latent fingerprints in these four images are shown in Fig. 12. These component fingerprints are matched by using COTS2 matcher against a background database consisting of 20 rolled fingerprint images from the two donors (one image per finger), 258 rolled fingerprint 


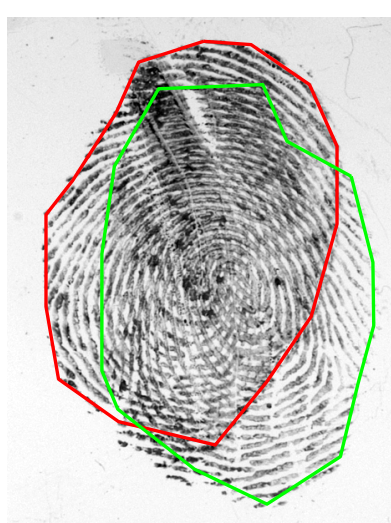

(a)

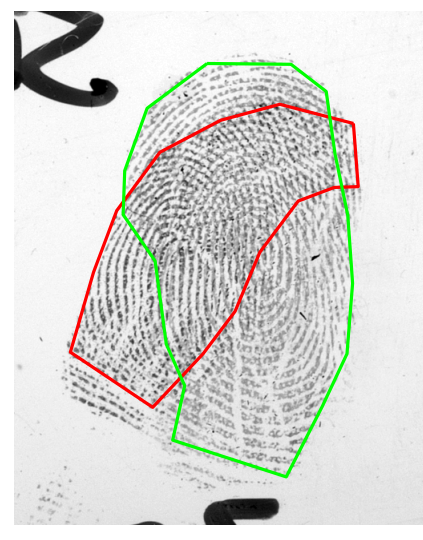

(b)

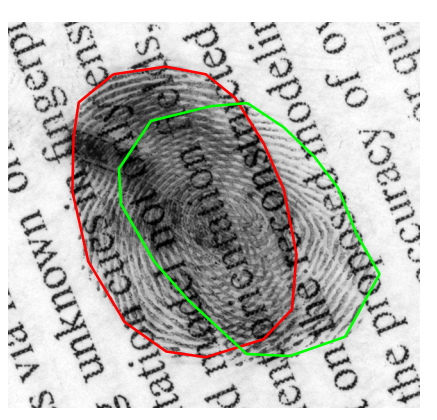

(c)

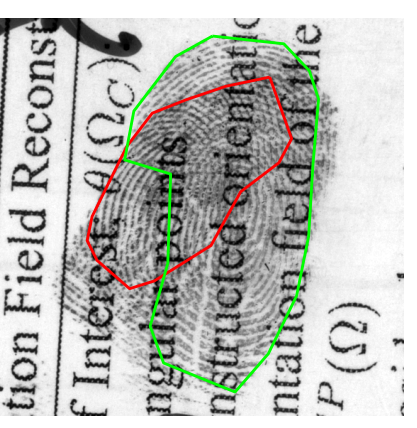

(d)

Fig. 11. Four images of real overlapping latents. The red and green polygons mark the first and second component latents, respectively.

TABLE II

MATCHING RESULTS OF COTS2 MATCHER ON THE COMPONENT LATENT FINGERPRINTS IN FIG. 11 AGAINST A BACKGROUND DATABASE OF 27,278 FULL FINGERPRINTS. THREE CASES ARE CONSIDERED: COMPONENT LATENTS WITHOUT SEPARATION, COMPONENT LATENTS SEPARATED BY THE RELAXATION LABELING BASED METHOD [4], AND COMPONENT LATENTS SEPARATED BY THE PROPOSED MODEL BASED METHOD.

\begin{tabular}{|l|l||l|l|l|l|l|l|}
\hline \multicolumn{2}{|c|}{$\begin{array}{c}\text { Component } \\
\text { Latents }\end{array}$} & \multicolumn{2}{c|}{ No Separation } & \multicolumn{2}{c|}{ Relaxation Labeling } & \multicolumn{2}{c|}{ Proposed } \\
\cline { 2 - 8 } & Score & Rank & Score & Rank & Score & Rank \\
\hline \multirow{3}{*}{ (a) } & $\# 1$ & 3025 & 12 & 3680 & 1 & 4040 & 1 \\
\cline { 2 - 8 } & $\# 2$ & 4286 & 1 & 2857 & 8 & 5897 & 1 \\
\hline \multirow{3}{*}{ (b) } & $\# 1$ & 4952 & 1 & 3084 & 23 & 7373 & 1 \\
\cline { 2 - 8 } & $\# 2$ & 3288 & 3 & 2781 & 19 & 4964 & 1 \\
\hline (c) & $\# 1$ & 1546 & 15956 & 1748 & 4430 & 2493 & 42 \\
\cline { 2 - 8 } & $\# 2$ & 1877 & 5243 & 1741 & 3245 & 3811 & 1 \\
\hline (d) & $\# 1$ & 2520 & 393 & 1642 & 5218 & 2939 & 23 \\
\cline { 2 - 8 } & $\# 2$ & 1480 & 19073 & 2100 & 779 & 2472 & 102 \\
\hline
\end{tabular}

images from NIST SD27, and the first 27,000 rolled fingerprint images from NIST SD14. The matching results, as reported in Table II, demonstrate that (i) poor quality overlapping latents (e.g. the second component latent in Fig. 11(c) and the first component latent in Fig. 11(d)), if properly separated, can provide valuable evidence for latent identification, and (ii) the proposed method is more robust on poor quality overlapping latents than the relaxation labeling based method.

\section{B. Comparison of Orientation Field Models}

As discussed in Section III.C, most existing orientation field models which are based on singular points are not suitable for latent fingerprints, and for the polynomial based models, different polynomial basis functions have been proposed. In this section, we compare the performance of the proposed separation algorithm when different polynomial basis functions are used, including monomials in [13], [14], [17], [18], Legendre polynomials in [15], and trigonometric polynomials (i.e. FOMFE) in [16], [19]. For all of the three types of polynomial basis functions, we set their order to four, which is the recommended optimal value by the authors of studies which proposed these polynomial basis function based models. In the experiments, we separate the four real overlapping latent fingerprints by using different polynomial basis functions, and use COTS2 matcher to match the separated component latents against the large background database used in the previous section. Although the Legendre polynomial based model and FOMFE were initially proposed without using singular points, for fair comparison in our experiments, we also apply them in the case of using singular points.

The retrieval ranks of the corresponding mated fingerprints of the latents are given in Table III. From these results, we can see that (i) all the three orientation field models benefit from the use of singular points, particularly for lower quality latents, and (ii) none of the three models consistently performs better than the others, but the monomial based model performs, on average, the best, especially when singular points are not used. Figure 13 shows the reconstructed orientation fields by the proposed method with monomial basis functions for the component latents in Fig. 11(c). The first component latent in Fig. 11(c) has such a poor quality that its core points can not be clearly located and only one possible delta close to its boundary is marked. The reconstructed orientation fields of this component latent (see Figs. 13(b) and 13(c)) show that the marked delta does not help much and can even degrade the matching performance for this very poor quality component latent. On the other hand, if the singular points can be reliably marked in a component latent, for example, for the second component latent in Fig. 11(c), the reconstructed orientation field is much more accurate when the reliable singular points are used (see Figs. 13(e) and 13(f)), and thus the matching accuracy of the separated component latent can be significantly 
(a)
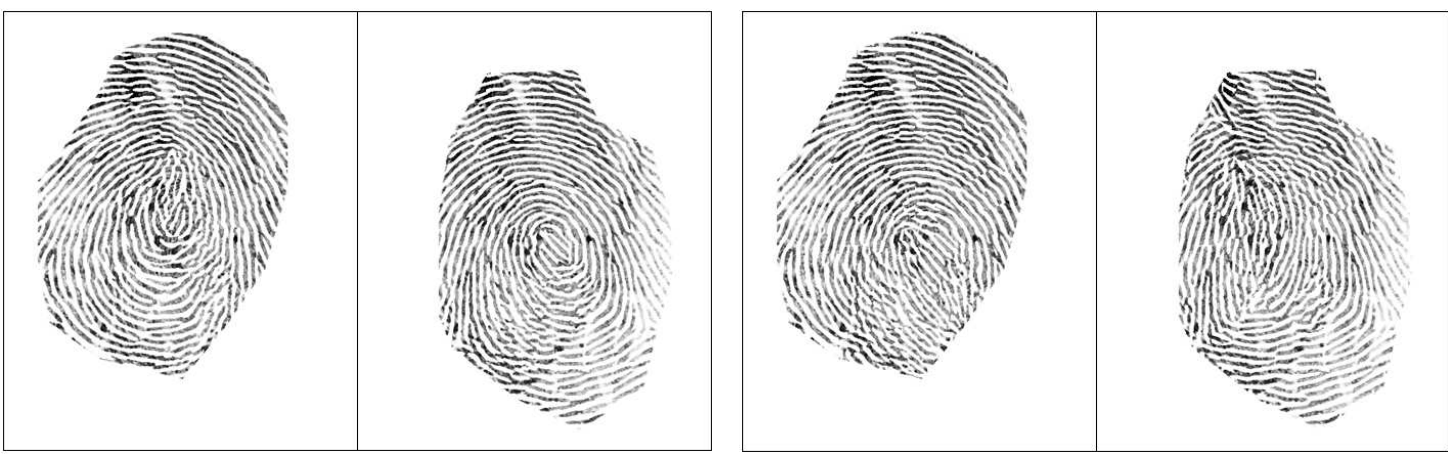

(b)
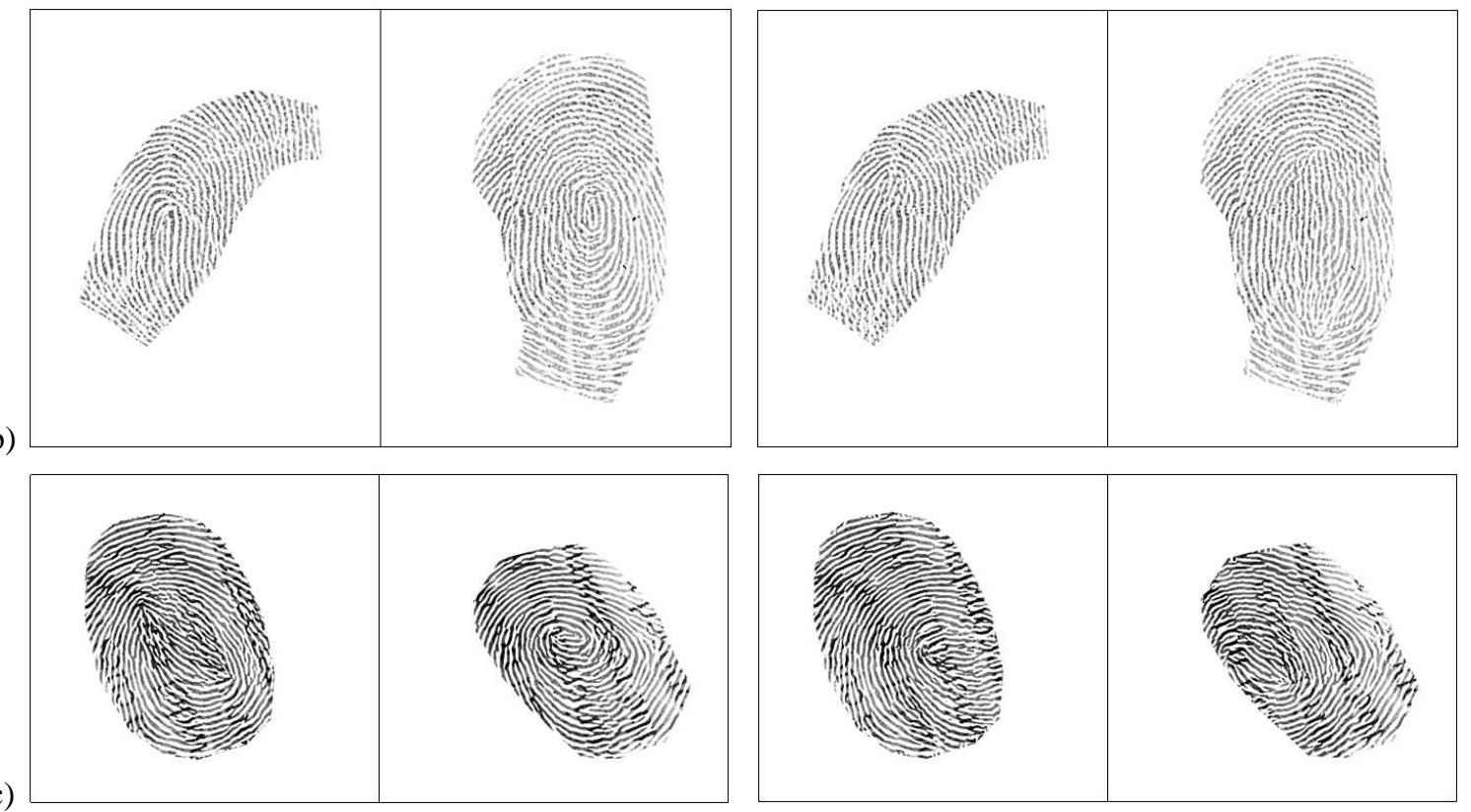

(c)
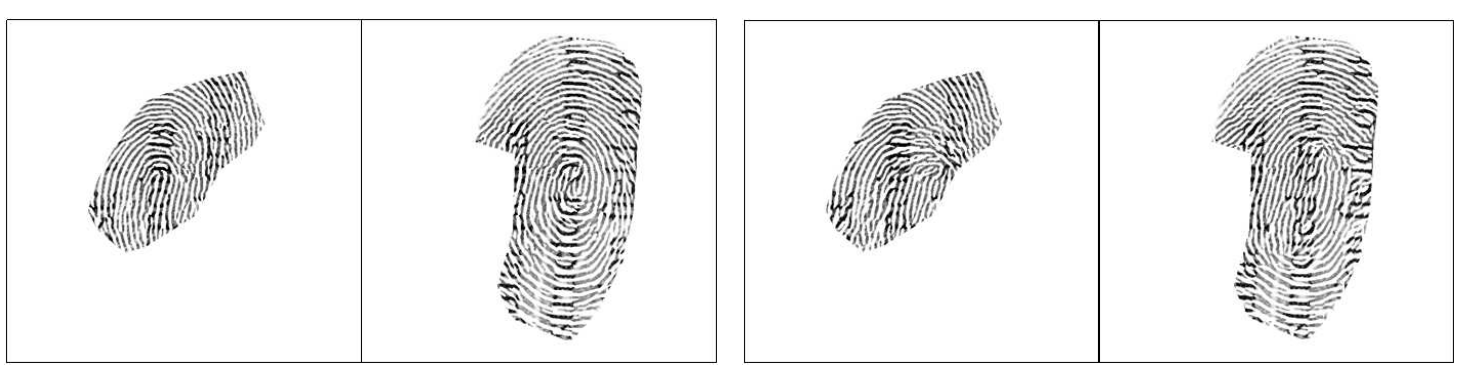

Fig. 12. Separation results of the real overlapping latents in Figs. 11(a)-11(d). The first and third columns show the $1^{s t}$ component latent fingerprint separated by the proposed method and the relaxation labeling based method, respectively. The second and fourth columns show the $2^{\text {nd }}$ component latent fingerprint separated by the proposed method and the relaxation labeling based method, respectively.

improved. Figure 14 compares the reconstructed orientation fields for the second component latent in Fig. 11(d) by using monomial based, Legendre polynomial based, and FOMFE models when the cues in Fig. 14(a) are provided. Although the unavailability of the core points impairs the accuracy of all the three models, the monomial based model gives the most accurate reconstruction of the orientation field.

\section{Impact of Markup Cues}

In the last section, we demonstrated the importance of manually marked singular points in orientation field reconstruction and overlapping fingerprints separation. In this section, we discuss the impact of manually marked orientation cues on the separation performance of the proposed model based method with monomial basis functions. The manually marked orientation cues are essentially seeds to the proposed model based separation method. It is thus important to provide a sufficient number of accurate orientation cues in order for a reasonable reconstruction of fingerprint orientation field. General intuition would suggest marking uniformly distributed orientation cues in fingerprints. Our experiments, however, show that some regions in fingerprints are more critical and providing orientation cues in such regions can significantly improve the reconstruction results. These regions are of high 
TABLE III

RETRIEVAL RANKS BY COTS2 MATCHER OF THE MATED FINGERPRINTS OF THE COMPONENT LATENT FINGERPRINTS (SEE FIG. 11) IN A BACKGROUND DATABASE OF 27,278 FULL FINGERPRINTS. THE SEPARATED COMPONENT LATENTS ARE OBTAINED BY USING THE PROPOSED METHOD WITH THREE DIFFERENT TYPES OF POLYNOMIAL BASIS FUNCTIONS: MONOMIALS, LEGENDRE POLYNOMIALS, AND FOMFE. ALL OF THE THREE TYPES OF POLYNOMIAL BASIS FUNCTIONS ARE APPLIED IN TWO CASES: USING SINGULAR POINTS (SP) AND NOT USING SINGULAR POINTS (NO SP).

\begin{tabular}{|l|l||l|l|l|l|l|l|}
\hline \multicolumn{2}{|c||}{$\begin{array}{c}\text { Component } \\
\text { Latents }\end{array}$} & \multicolumn{2}{c|}{ Monomials } & \multicolumn{2}{c|}{ Legendre Polynomials } & \multicolumn{2}{c|}{ FOMFE } \\
\cline { 2 - 8 } & SP & No SP & SP & No SP & SP & No SP \\
\hline \multirow{2}{*}{ (a) } & $\# 1$ & 1 & 1 & 1 & 54 & 1 & 5 \\
\cline { 2 - 8 } & $\# 2$ & 1 & 3 & 1 & 1 & 1 & 2 \\
\hline (b) & $\# 1$ & 1 & 1 & 1 & 1 & 1 & 1 \\
\cline { 2 - 8 } & $\# 2$ & 1 & 4 & 1 & 19 & 1 & 178 \\
\hline (c) & $\# 1$ & 42 & 2 & 77 & 2789 & 1 & 9 \\
\cline { 2 - 8 } & $\# 2$ & 1 & 38 & 1 & 825 & 1 & 1096 \\
\hline (d) & $\# 1$ & 23 & 1856 & 90 & 1446 & 28 & 53 \\
\cline { 2 - 8 } & $\# 2$ & 102 & 686 & 2351 & 2315 & 2734 & 17185 \\
\hline
\end{tabular}

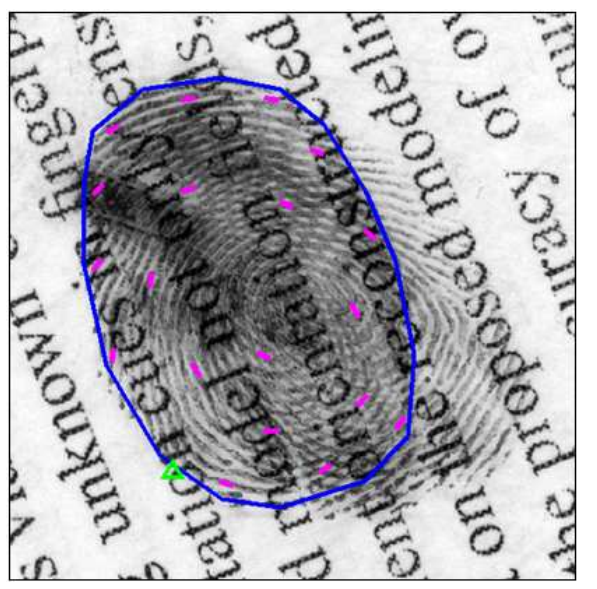

(a)

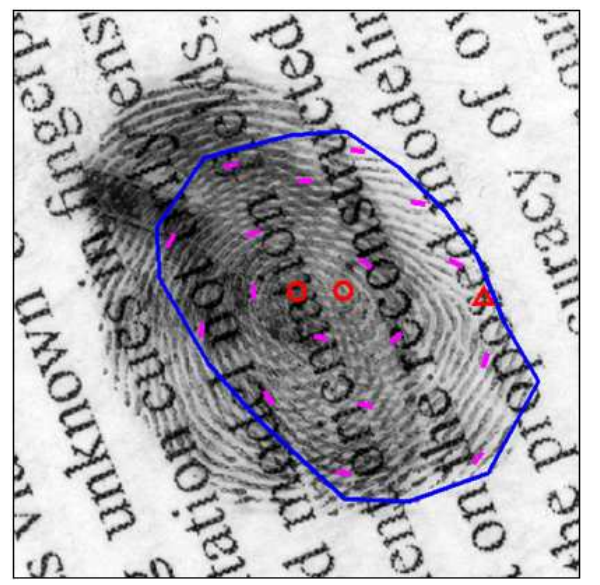

(d)

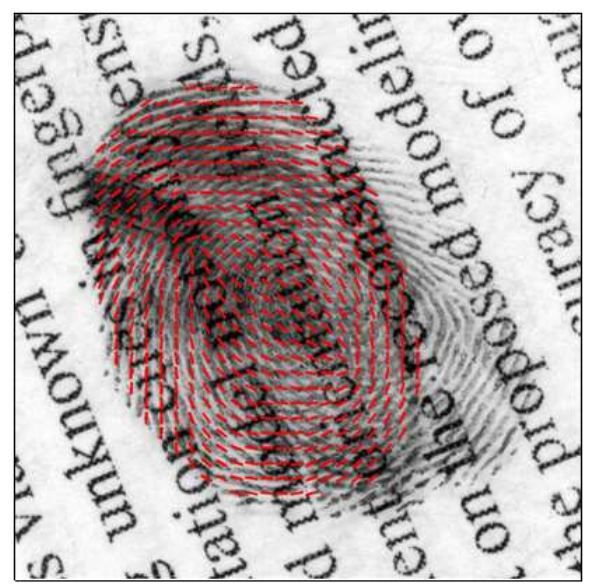

(b)

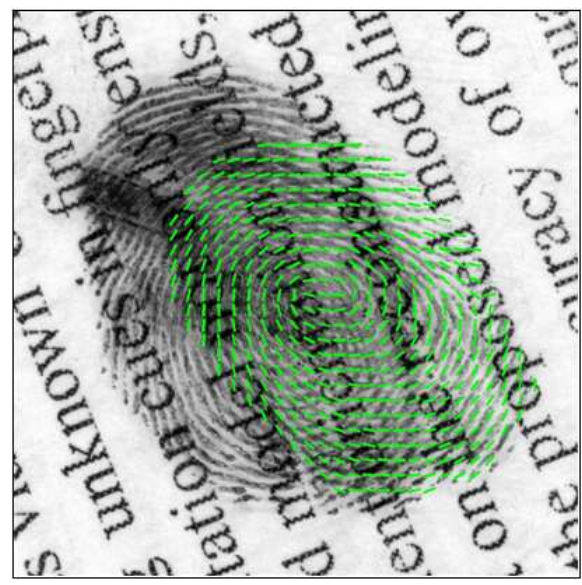

(e)

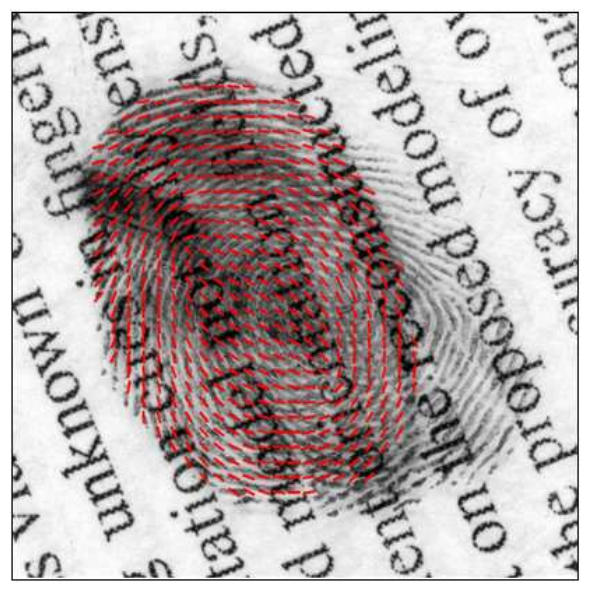

(c)

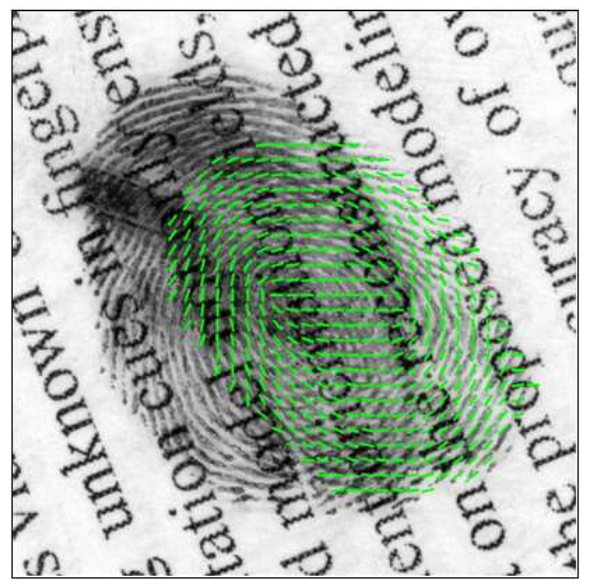

(f)

Fig. 13. Impact of singular points. (a) and (d) show the manually marked ROI, singular points, and orientation cues in the two component latents in Fig. 11(c). (b) and (c) are the first component latent separated by the proposed algorithm with monomial basis functions, and (e) and (f) are the second component latent. Singular points are used for (b) and (e), but not used for (c) and (f). 


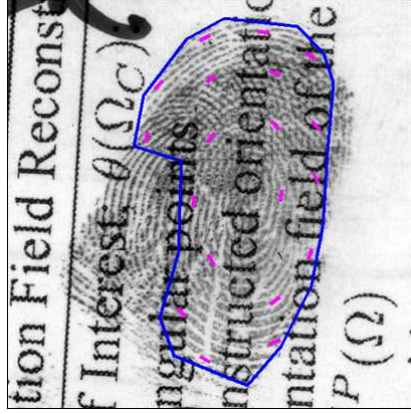

(a)

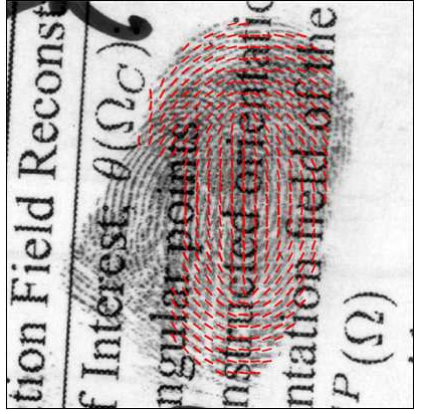

(b)

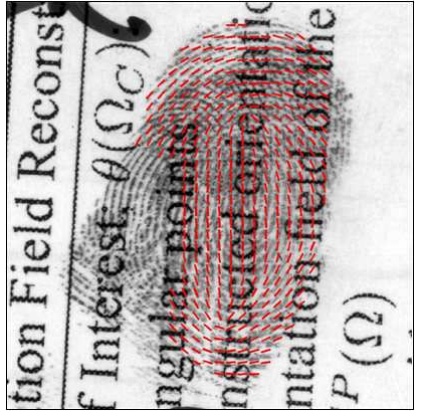

(c)

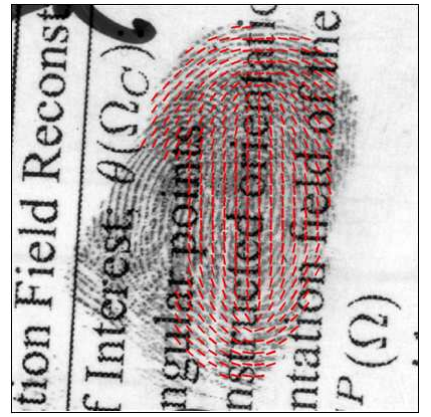

(d)

Fig. 14. Comparison between orientation field models. (a) Manually marked ROI and orientation cues in the second component latent in Fig. 11(d). Singular points are not marked. (b), (c) and (d) are, respectively, the reconstructed orientation fields using monomial based, Legendre polynomial based, and FOMFE models. By using the COTS2 matcher, the scores between the corresponding separated component latents and the mated rolled fingerprint are 2061, 1817, and 1327 , respectively.

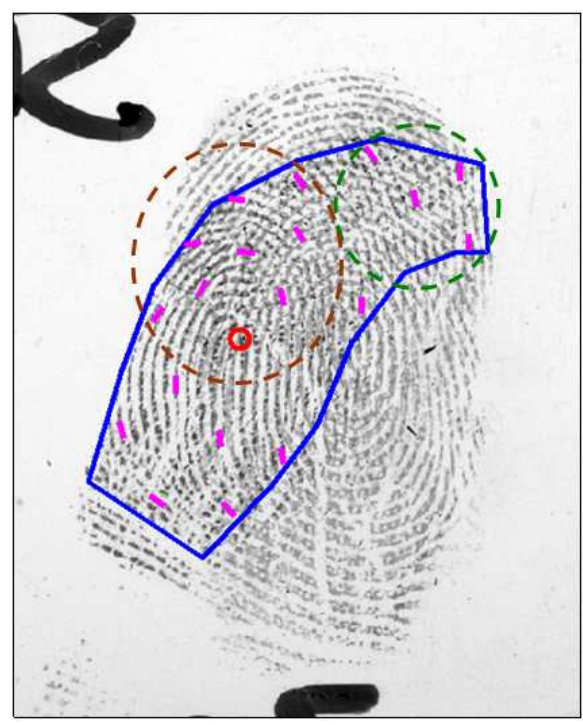

(a)

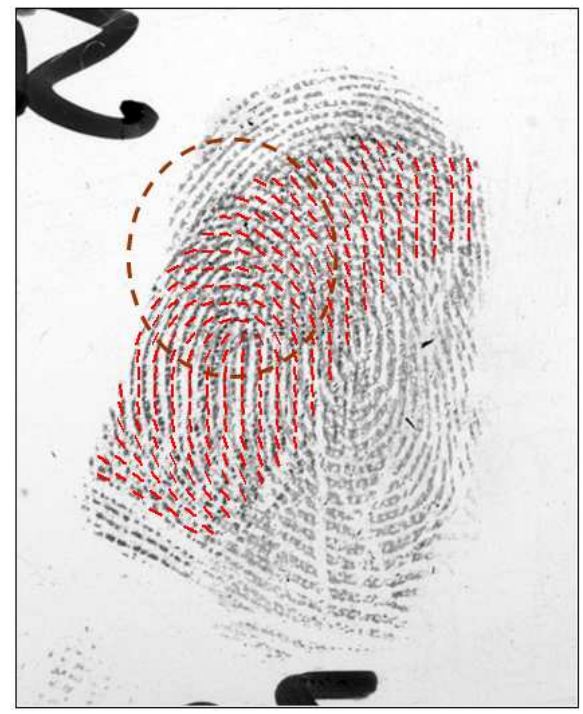

(d)

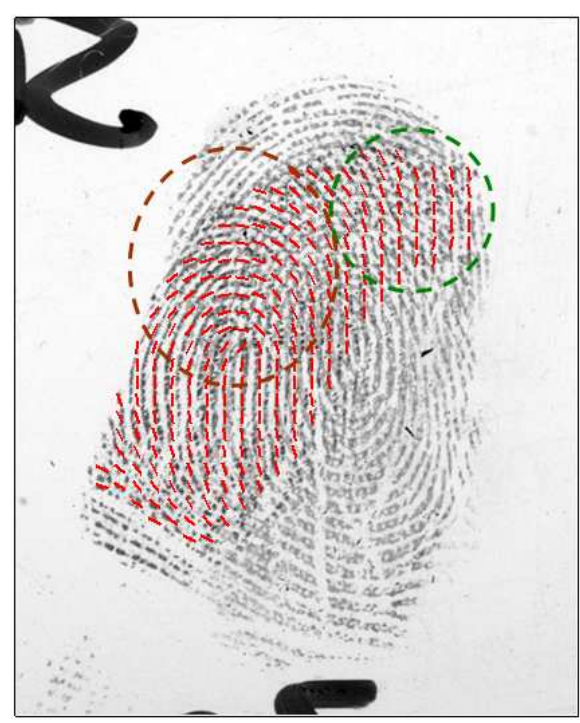

(b)

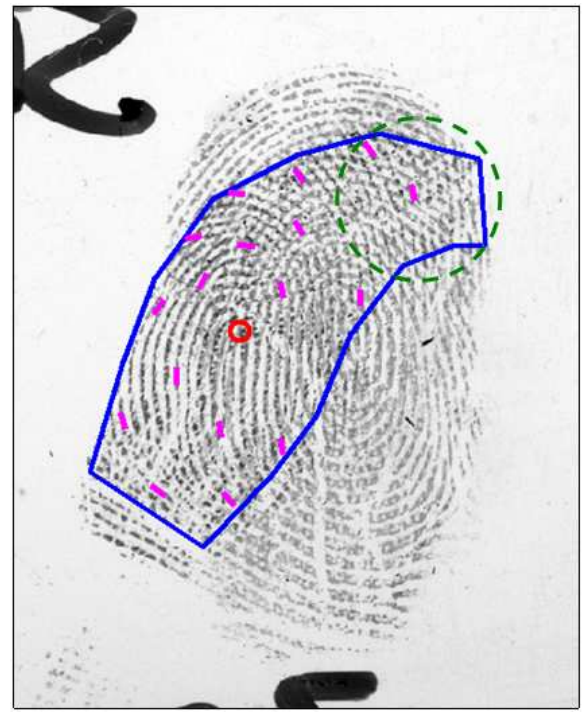

(e)

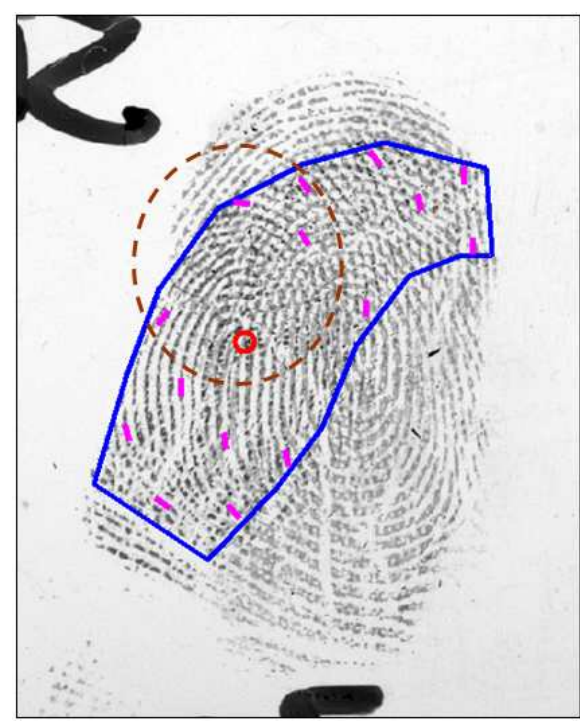

(c)

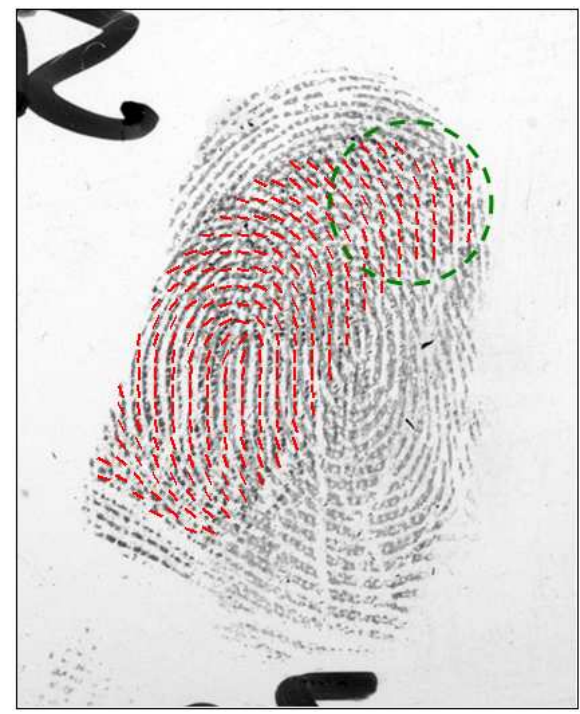

(f)

Fig. 15. Impact of orientation cues. (a) Manually marked ROI, singular points, and orientation cues in the first component latent of Fig. 11(b), (b) reconstructed orientation field in (b) (dashed brown and green circles highlight, repsectively, a region of high curvature and a region far from singular points), (c) a small number of orientation cues in the high curvature region are removed, (d) the corresponding reconstructed orientation field, (e) a small number of orientation cues in the region far from singular points are removed, and (f) the corresponding reconstructed orientation field. 


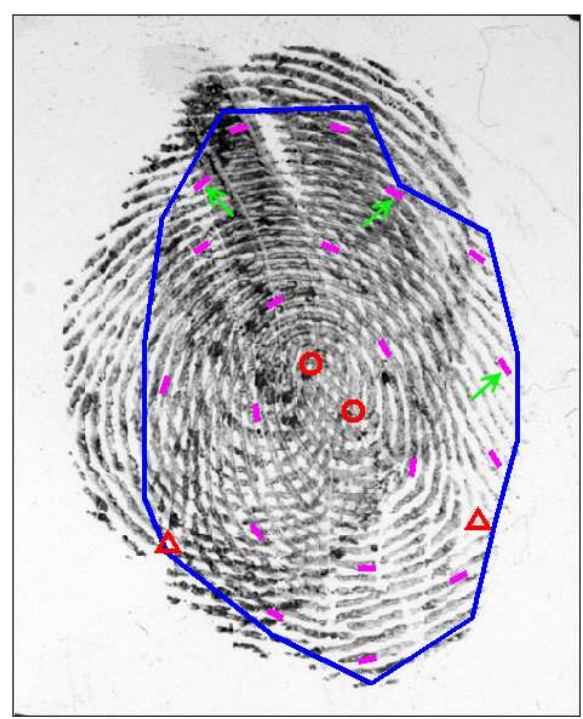

(a)

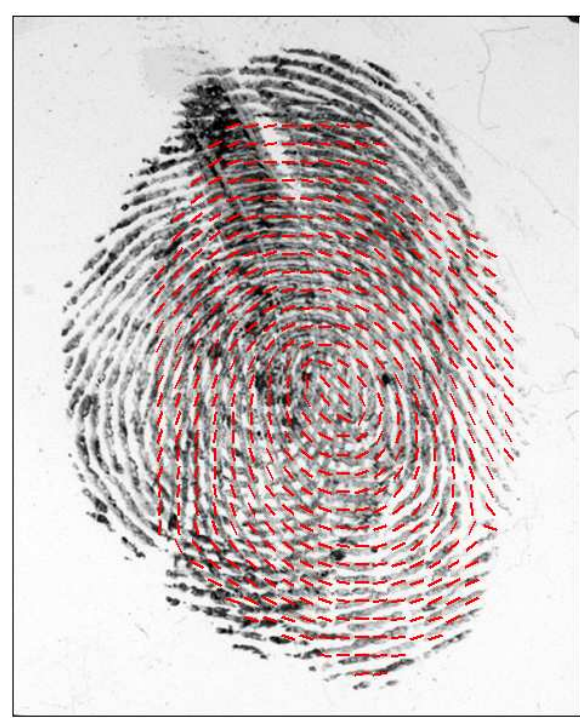

(b)

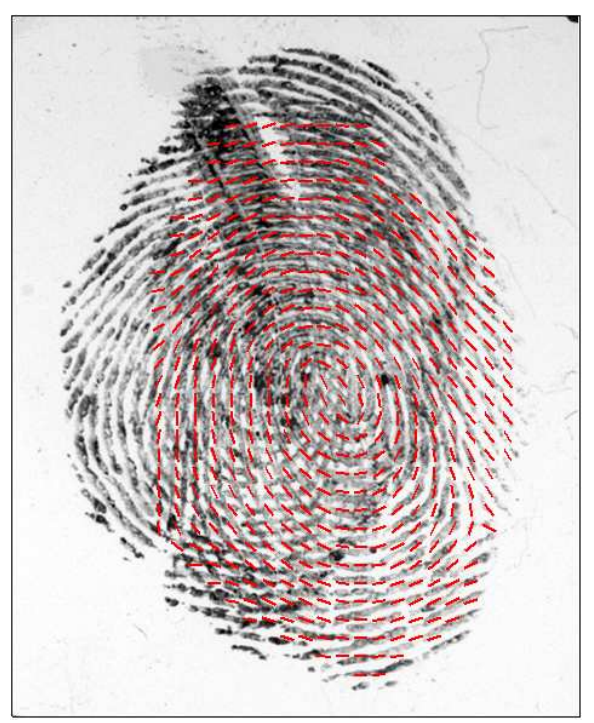

(c)

Fig. 16. Impact of orientation cues. (a) Manually marked ROI, singular points, and orientation cues in the second component latent in Fig. 11(a), used for reconstructing the orientation field in (b) and the separated component latent in Fig. 12. (c) shows the reconstructed orientation field when some cues near the boundary (see the cues pointed to by arrows) are removed.

curvature, regions far from singular points, and regions close to boundary. Next, we demonstrate this by using the first component latent in Fig. 11(b) and the second component latent in Fig. 11(a).

Figure 15(a) highlights two critical regions in the first component latent in Fig. 11(b): one of them has high curvature and the other is far from singular points. We remove some of the orientation cues from the high curvature region (Fig. 15(c)) and from the region far from singular points (Fig. 15(e)), respectively. The corresponding reconstructed orientation fields are shown in Figs. 15(d) and 15(f). It can be seen from these figures that the reconstructed orientation fields, after discarding the orientation cues in the two regions, can not accurately follow the fingerprint ridges. The genuine match scores of COTS2 matcher in these two cases (Fig. 15(c) and Fig. 15(e)) are, respectively, 5797 and 5186, which are lower than the score (7373) obtained when those orientation cues were not excluded (Fig. 15(a)).

Figure 16 shows the results on another example, i.e. the second component latent in Fig. 11(a). Figure 16(a) shows the cues based on which the separated component latent in Fig. 12(a) is obtained, and the corresponding reconstructed orientation field is shown in Fig. 16(b). If we remove some of the cues near the boundary as marked by the arrows in Fig. 16(a), the reconstructed orientation field becomes less accurate as shown in Fig. 16(c). This is due to the insufficient number of constraints (i.e. markup cues) on the boundary. Prediction of orientations on the boundary is more like extrapolation (particularly when insufficient cues are given), which is more difficult than interpolation. Because of the less accurate reconstructed orientation field, the genuine match scores of COTS2 matcher for the separated component latents also decreases from 5897 to 4880 .

Based on the above results, it is suggested that the latent examiners provide sufficient number of orientation cues in regions of high curvature and in regions far from singular points or close to boundary. Although in practice, it could be difficult for the latent examiner to decide at the first glance how many cues will be sufficient for an accurate separation of overlapping latents, we believe that the difficult task of separating overlapping latents should be viewed as a semiautomatic and iterative task rather than a fully automatic or "lights out" task. In this sense, the model based separation method proposed here provides a very useful interactive tool for latent examiners.

\section{Computational Complexity}

The proposed separation method involves an iterative process in the reconstruction of the orientation field of overlapping fingerprints (see Algorithm 1). The time required by this process depends on the size of the fingerprint, the number of given orientation cues, and the precision (i.e. block size) of the estimated orientation field. According to our experimental results, for 500 ppi fingerprint images and a block size of $8 \times 8$ pixels (as used in our experiments), the proposed algorithm converges in about five iterations and takes less than one second (on a $3 \mathrm{GHz}$ processor with $4 \mathrm{~GB}$ memory) to reconstruct the orientation field per component fingerprint. As a comparison, according to [4], the relalaxtion labeling based method in [4] takes about three seconds to separate the orientation fields in one overlapped fingerprint image, and the method in [2] takes more than 30 seconds. However, the proposed method requires additional manually marked features, i.e. orientation cues. Although marking these additional cues does take extra time from latent examiners, these cues enable the proposed method to more accurately separate low quality overlapping fingerprints.

It is worthwhile to mention that while it is also possible 
to incorporate manually marked orientation cues into the relaxation labeling based methods, this would require latent examiners to check the two dominant orientations in the overlapping area blocks. This is because the relaxation labeling algorithm is not able to correct the errors in the estimated orientation field or to handle the discrepancy between markup orientation cues and computed local orientations ${ }^{1}$. What the relaxation labeling algorithm does is to simply classify the given two orientations in a block to one of the two component fingerprints based on local consistency of the fingerprint orientation field. On the contrary, the proposed model based method can not only predict unknown orientations, but it can also fix the errors in the estimated orientation field. As a result, the proposed method can more effectively as well as efficiently utilize the orientation cues manually marked by latent examiners in the reconstruction of the component orientation fields in overlapping fingerprints.

\section{Conclusions}

Overlapping latent fingerprints are frequently encountered at crime scenes. The identification of component fingerprints, both by latent examiners and by AFIS, is very challenging because of the complex background, poor quality, and contaminated ridge structures. In this paper, we have proposed a model based method for separating overlapping latent fingerprints. The proposed algorithm reconstructs the orientation field of overlapping fingerprints based on a set of manually marked features, including regions of interest, singular points, and orientation cues. Based on the underlying model of fingerprint ridge orientation field, the proposed method can simultaneously predict unknown orientations in fingerprints and regularize the estimated orientations. Experimental results on both simulated and real overlapping latents demonstrate that the proposed method, compared to state-of-the-art relaxation labeling based method, is more effective, thanks to the manually marked cues and the underlying orientation field models.

Fingerprint orientation field models have been widely used for regularizing the estimation of fingerprint ridge orientation field. In this paper, we employed the models not just for regularization, but also for predicting unknown orientations. Although we have primarily utilized the combination model based on the Zero-Pole model and monomial basis functions [13], [17], [18], which requires singular points, and the Legendre polynomial based [15] and FOMFE [16], [19] models, which do not need singular points, our approach does not depend on nor require any specific orientation field model. In our future work, we plan to improve the prediction capability of existing fingerprint orientation field models in order to further improve the accuracy of model based separation of overlapping latent fingerprints. Another direction we plan to take is to enhance the feasibility of the proposed method by studying the optimal way of specifying orientation cues. One potential way to guide the specification of orientation cues is to divide fingerprints into different regions. From this perspective, more quantitative and systematic evaluation of the

\footnotetext{
${ }^{1}$ It is for this reason that the authors in [4] included a smoothing operation to the separated orientation fields to eliminate some errors.
}

impact of orientation cues in different fingerprint regions on a large database will be very useful.

\section{ACKNOWLEDGEMENT}

Anil Jain's research was supported in part by the WCU (World Class University) program through the National Research Foundation of Korea funded by the Ministry of Education, Science and Technology (R31-2008-000-1000-0). The authors would like to thank Capt. Gregoire P. Michaud, D/Lt. Gary S. Daniels, D/Sgt. Scott Hrcka, and D/Sgt. Derek Emme from Michigan State Police and Soweon Yoon from Michigan State University for their support in data collection and useful discussion. All correspondence should be directed to Anil K. Jain.

\section{REFERENCES}

[1] D. Maltoni, D. Maio, A. K. Jain, and S. Prabhakar, Handbook of Fingerprint Recognition (Second Edition). Springer-Verlag, 2009.

[2] F. Chen, J. Feng, A. K. Jain, J. Zhou, and J. Zhang, "Separating overlapped fingerprints," IEEE Transactions on Information Forensics and Security, vol. 6, no. 2, pp. 346-359, 2011.

[3] M. D. Garris and R. M. McCabe, "NIST Special Database 27: Fingerprint Minutiae from Latent and Matching Tenprint Images," June 2000, http://www.nist.gov/srd/nistsd27.cfm.

[4] Y. Shi, J. Feng, and J. Zhou, "Separating overlapped fingerprints using constrained relaxation labeling," in Proc. International Joint Conference on Biometrics, 2011.

[5] X. Fan, D. Liang, and L. Zhao, "A scheme for separating overlapped fingerprints based on partition mask," Computer Engineering and Applications, vol. 40, no. 2, pp. 80-81, 2004 (in Chinese).

[6] R. Geng, Q. Lian, and M. Sun, "Fingerprint separation based on morphological component analysis," Computer Engineering and Applications, vol. 44, no. 16, pp. 188-190, 2008 (in Chinese).

[7] M. Singh, D. Singh, and P. Kalra, "Fingerprint separation: An application of ICA," in Proc. SPIE, Mobile Multimedia/Image Processing, Security, and Applications, vol. 6982, 2008, pp. 69820L-1-69 820L-11.

[8] D. Maio, D. Maltoni, R. Cappelli, J. L. Wayman, and A. K. Jain, "FVC2002: Second fingerprint verification competition," in Proc. International Conference on Pattern Recognition, vol. 3, 2002, pp. 811-814.

[9] L. Hong, Y. Wan, and A. K. Jain, "Fingerprint image enhancement: Algorithm and performance evaluation," IEEE Transactions on Pattern Analysis and Machine Intelligence, vol. 20, no. 8, pp. 777-789, 1998.

[10] B. G. Sherlock and D. M. Monro, "A model for interpreting fingerprint topology," Pattern Recognition, vol. 26, no. 7, pp. 1047-1055, 1993.

[11] A. K. Jain and J. Feng, "Latent fingerprint matching," IEEE Transactions on Pattern Analysis and Machine Intelligence, vol. 33, no. 1, pp. 88-100, 2011.

[12] M. Kass and A. Witkin, "Analyzing oriented patterns," Computer Vision, Graphics, and Image Processing, vol. 37, no. 3, pp. 362-385, 1987.

[13] J. Zhou and J. Gu, "A model-based method for the computation of fingerprints orientation field," IEEE Transactions on Image Processing, vol. 13 , no. 6 , pp. 821-835, 2004.

[14] J. Gu, J. Zhou, and D. Zhang, "A combination model for orientation field of fingerprints," Pattern Recognition, vol. 37, pp. 543-553, 2004.

[15] S. Ram, H. Bischof, and J. Birchbauer, "Modelling fingerprint ridge orientation using legendre polynomials," Pattern Recognition, vol. 43, pp. 342-357, 2010.

[16] Y. Wang, J. Hu, and D. Phillips, "A fingerprint orientation model based on $2 \mathrm{~d}$ Fourier expansion (FOMFE) and its application to singularpoint detection and fingerprint indexing," IEEE Transactions on Pattern Analysis and Machine Intelligence, vol. 29, no. 4, pp. 573-585, 2007.

[17] S. Yoon, J. Feng, and A. K. Jain, "Latent fingerprint enhancement via robust orientation field estimation," in Proc. International Joint Conference on Biometrics, 2011.

[18] J. Feng and A. K. Jain, "Fingerprint reconstruction: From minutiae to phase," IEEE Transactions on Pattern Analysis and Machine Intelligence, vol. 33, no. 2, pp. 209-223, 2011.

[19] Y. Wang and J. Hu, "Global ridge orientation modeling for partial fingerprint identification," IEEE Transactions on Pattern Analysis and Machine Intelligence, vol. 33, no. 1, pp. 72-87, 2011. 
[20] A. Ross, J. Shah, and A. K. Jain, "From template to image: Reconstructing fingerprints from minutiae points," IEEE Transactions on Pattern Analysis and Machine Intelligence, vol. 29, no. 4, pp. 544-560, 2007.

[21] R. Cappelli, A. Lumini, D. Maio, and D. Maltoni, "Fingerprint image reconstruction from standard templates," IEEE Transactions on Pattern Analysis and Machine Intelligence, vol. 29, no. 9, pp. 1489-1503, 2007.

[22] P. Vizcaya and L. Gerhardt, "A nonlinear orientation model for global description of fingerprints," Pattern Recognition, vol. 29, no. 7, pp. 1221-1231, 1996.

[23] J. Zhou and J. Gu, "Modeling orientation fields of fingerprints with rational complex functions," Pattern Recognition, vol. 37, pp. 389-391, 2004.

[24] J. Li, W.-Y. Yau, and H. Wang, "Constrained nonlinear models of fingerprint orientations with prediction," Pattern Recognition, vol. 39, pp. 102-114, 2006.

[25] J. Li, W.-Y. Yau, H. Wang, and W. Ser, "Stability analysis of constrained nonlinear phase portrait models of fingerprint orientation images," in Proc. International Conference on Biometrics, 2007.

[26] S. Huckemann, T. Hotz, and A. Munk, "Global models for the orientation field of fingerprints: An approach based on quadratic differentials," IEEE Transactions on Pattern Analysis and Machine Intelligence, vol. 30, no. 9, pp. 1507-1519, 2008.

[27] S. C. Dass, "Markov random field models for directional field and singularity extraction in fingerprint images," IEEE Transactions on Image Processing, vol. 13, no. 10, pp. 1358-1367, 2004.

[28] K. Lee and S. Prabhakar, "Probabilistic orientation field estimation for fingerprint enhancement and verification," in Proc. Biometric Symposium, 2008.

[29] C. I. Watson, "NIST Special Database 14: Mated Fingerprint Card Pairs 2," June 2000, http://www.nist.gov/srd/nistsd14.cfm.

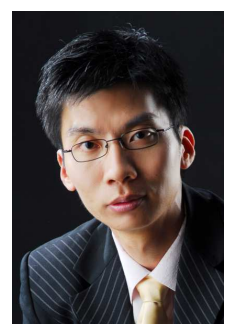

Qijun Zhao is a post-doc fellow in the Department of Computer Science and Engineering at Michigan State University, East Lansing. He received his B.S. and M.S. degrees from the Department of Computer Science and Technology, Shanghai Jiao Tong University, respectively in 2003 and 2006, and his Ph.D. degree in 2010 from the Department of Computing, The Hong Kong Polytechnic University. His research interests mainly lie in the fields of pattern recognition, machine learning, image proto biometrics, information security, and intelligent systems. cessing, and artificial intelligence, with applications

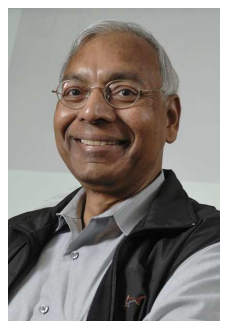

Anil K. Jain (S'70-M'72-SM'86-F'91) is a university distinguished professor in the Department of Computer Science and Engineering at Michigan State University, East Lansing. His research interests include pattern recognition and biometric authentication. He served as the editor-in-chief of the IEEE TRANSACTIONS ON PATTERN ANALYSIS AND MACHINE INTELLIGENCE (19911994). The holder of six patents in the area of fingerprints, he is the author of a number of book$\mathrm{s}$, including Handbook of Fingerprint Recognition (2009), Handbook of Biometrics (2007), Handbook of Multibiometrics (2006), Handbook of Face Recognition (2005), BIOMETRICS: Personal Identification in Networked Society (1999), and Algorithms for Clustering Data (1998). He served as a member of the Defense Science Board and The National Academies committees on Whither Biometrics and Improvised Explosive Devices. Dr. Jain received the 1996 IEEE TRANSACTIONS ON NEURAL NETWORKS Outstanding Paper Award and the Pattern Recognition Society best paper awards in 1987, 1991, and 2005. He is a fellow of the AAAS, ACM, IAPR, IEEE, and SPIE. He has received Fulbright, Guggenheim, Alexander von Humboldt, IEEE Computer Society Technical Achievement, IEEE Wallace McDowell, ICDM Research Contributions, and IAPR King$\mathrm{Sun} \mathrm{Fu}$ awards. ISI has designated him a highly cited researcher. According to Citeseer, his book Algorithms for Clustering Data (Englewood Cliffs, NJ: Prentice-Hall, 1988) is ranked \#93 in most cited articles in computer science. 